Preprint USM-TH-85

\title{
The Magnetized Electron Gas in terms of Hurwitz Zeta Functions
}

\author{
Claudio O. Dib円 and Olivier Espinosa \\ Departamento de Física \\ Universidad Técnica Federico Santa María \\ Casilla 110-V \\ Valparaíso, Chile
}

\begin{abstract}
We obtain explicit expressions for thermodynamic quantities of a relativistic degenerate free electron gas in a magnetic field in terms of Hurwitz Zeta functions. The formulation allows for systematic expansion in all regimes. Three energy scales appear naturally in the degenerate relativistic gas: the Fermi energy $E_{\mathrm{F}}$, the temperature $T$ and an energy related to the magnetic field or Landau level spacing, $e B / E_{\mathrm{F}}$. We study the cold and warm scenarios, $T \ll e B / E_{\mathrm{F}}$ and $e B / E_{\mathrm{F}} \ll T$, respectively. We reproduce the oscillations of the magnetization as a function of the field in the cold regime and the dilution of them in the warm regime.
\end{abstract}

Corresponding Author:

Olivier Espinosa (espinosa@fis.utfsm.cl)

Departamento de Física, Universidad Técnica Federico Santa María

Casilla 110-V, Valparaíso, Chile.

Phone: $+56(32) 654-506$

Fax: $+56(32) 797-656$

PACS: 05.30Fk, 71.10Ca, 02.30Gp, 97.60Jd.

Keywords: Electron Gas, Landau Levels, Magnetism, Hurwitz Zeta Function.

\footnotetext{
${ }^{1}$ cdib@fis.utfsm.cl

2 espinosa@fis.utfsm.cl
} 


\section{Introduction}

The study of the thermodynamic properties of degenerate relativistic electron gases in strong magnetic fields, as those found in compact astrophysical objects, was started long ago [1]. Although in that work the problem was stated in quite general terms, that is, arbitrary temperature and relativistic electrons described by Dirac's equation, approximate analytical results were obtained only in the limits of low temperatures and non-relativistic electrons. While the nonrelativistic approximation has some validity in white dwarfs, it is inappropriate for the electron gas existing inside neutron stars. In fact, in a typical neutron star the electron density is of the order of $10^{-2} \mathrm{fm}^{-3}$, which implies a Fermi kinetic energy $T_{\mathrm{F}} \equiv E_{\mathrm{F}}-m$ of the order of $100 \mathrm{MeV}$. The temperature, on the other hand, is at most a few $\mathrm{MeV}$, which makes the ratio $T / T_{\mathrm{F}}$ a small parameter and the gas quite degenerate. The relativistic case was partially considered later on in Refs. 2, 3] with regard to the problem of self-magnetization, and in Ref. [1] in connection with the magnetic susceptibility. In all these works only the dominant contributions relevant for each case were kept in the analysis.

More recently, a renewed interest in the relativistic magnetized electron gas, both at zero and finite temperature, has appeared from the point of view of quantum field theory [5 9]. In these studies most of the emphasis is put on the formal aspects of the problem. For instance, in Ref. [7] an exact analytic expression is obtained for the effective action (also called the grand potential in statistical mechanics), which corrects an incomplete result given earlier [5] that had missed the de Haas - van Alphen oscillations that are present at low temperatures, exactly as in the non-relativistic case. In Ref. [8] the quantum field theory results of Ref. [7] are recast, for the case of low temperatures, in terms of sums over filled Landau levels, which is actually the starting point of the quantum statistical mechanical calculation developed in the earlier works 近].

In this work we revisit the usual elementary statistical mechanical approach, originally carried out by Landau [10,11 (see also Ref. [12]) for a non-relativistic gas, to study a highly degenerate relativistic free electron gas, in the presence of a uniform magnetic field. Starting from a closed analytical expression for the density of states for this system, we derive explicit, simple analytical expressions for the various quantities of thermodynamic interest, such as the density and magnetization. The object of central interest will be the grand potential, from which all relevant thermodynamic quantities can be computed.

It is well known that for a relativistic electron gas the Coulomb interactions among electrons and between the electrons and any background of positive charge that should exist in a neutral system are small (order $\alpha$ ) corrections to the kinetic energy, so we will neglect them. We will therefore work consistently to zeroth order in QED corrections. In particular, this means that we will take the electron gyromagnetic ratio exactly equal to two, $g=2$. Additionally, since we shall be solely concerned with the degenerate regime, we neglect the 
contribution of positrons altogether.

We will show that the grand potential at $T=0$ has the integral representation

$$
\Omega_{0}\left(E_{\mathrm{F}}, B\right)=-\frac{V}{4 \pi^{2}}(2 e B)^{5 / 2} \int_{0}^{\left(E_{\mathrm{F}}^{2}-m^{2}\right) / 2 e B} \frac{\mathcal{H}_{-1 / 2}(q)}{\sqrt{m^{2}+2 e B q}} d q,
$$

where $m$ and $e$ are the electron mass and the fundamental charge, respectively (we use natural units, so that $\hbar=c=k_{B}=1$ ). Consequently the electron density at $T=0$ is

$$
n_{0}\left(E_{\mathrm{F}}, B\right)=\frac{1}{2 \pi^{2}}(2 e B)^{3 / 2} \mathcal{H}_{-1 / 2}\left(\frac{E_{\mathrm{F}}^{2}-m^{2}}{2 e B}\right) .
$$

The function $\mathcal{H}_{-1 / 2}(q)$ appearing in both (11) and (2), is a combination of Hurwitz zeta functions, $\mathcal{H}_{-1 / 2}(q) \equiv \zeta\left(-\frac{1}{2},\{q\}\right)-\zeta\left(-\frac{1}{2}, q+1\right)-\frac{1}{2} \sqrt{q}$, and plays a pervading role in our study of the magnetized free electron gas. Since the pressure is given by $P=-\Omega / V$, the expressions (11) and (2) furnish a parametric representation of the equation of state at $T=0, P_{0}=P_{0}\left(E_{\mathrm{F}}, B\right), n_{0}=n_{0}\left(E_{\mathrm{F}}, B\right)$.

The expressions given above correspond to zero temperature, but, as we show later in the paper, for a system of non-interacting fermions the full finite temperature grand potential can be obtained from its zero temperature counterpart. For instance, in the degenerate regime finite temperature corrections are usually obtained systematically from the ground state quantities in the form of a Taylor series in powers of $T / \mu$, where $\mu$ is the chemical potential. Take, for example, $\Omega(T, \mu)$. As is well known, the leading correction to the zero-temperature result generally goes as $\left(T / E_{\mathrm{F}}\right)^{2}$. This will actually be the case, provided the grand potential at $T=0, \Omega_{0}\left(E_{\mathrm{F}}\right)$, does not vary greatly when $E_{\mathrm{F}}$ is changed by an amount of order $T$. However, as we will see, in our case $\Omega_{0}\left(E_{\mathrm{F}}\right)$ has contributions that oscillate rapidly with $E_{\mathrm{F}}$ due to the filling of discrete Landau levels. Therefore the approximation above is not valid, except for very low temperatures ( $T \ll e B / E_{\mathrm{F}}$, as we will see later). We will compute with our formalism the correct finite temperature behavior of $\Omega(T, \mu)$ and reproduce the previously known result that the oscillations are smoothed out as the temperature increases.

The novelty of our approach is the use of the Hurwitz Zeta function to deal with the discrete sums over Landau levels that accommodate the electrons in the presence of a uniform magnetic field. The traditional approach 12 makes use of the so-called Poisson summation formula which is of limited use if one needs to expand the resulting expressions in different regimes, such as for small fields. The Hurwitz zeta function approach is much more powerful in this case, as it leads to closed analytical expressions, which can eventually be evaluated numerically with ease, and is suitable for expansion in any desired regime. Although this is not the first appearance of the Hurwitz zeta function in connection 
with the system being studied [6, 9], this seems to be the first time that its analytic properties are fully put into use to unravel the thermodynamics of the relativistic magnetized free electron gas.

The Hurwitz zeta function $\zeta(z, q)$ is defined as the analytic extension to the whole complex $z$ plane of the series

$$
\zeta(z, q)=\sum_{n=0}^{\infty} \frac{1}{(n+q)^{z}}
$$

valid for $\operatorname{Re} z>1$ and $q \neq 0,-1,-2, \cdots$. The resulting function is analytic everywhere except at $z=1$, where it has a simple pole with unit residue (see Appendix A for details).

In section 2 we formulate the density of states in its most general form in terms of Hurwitz zeta functions. In section 3 we classify the physical regimes we want to study. The core of the thermodynamics is presented in Section 4 and particular studies of the magnetization are shown in Section 5. Details of the calculations and mathematical formulas are given in the appendices.

\section{The Density of States in terms of Hurwitz Functions}

The stationary states of a Dirac electron that moves in a uniform magnetic field $\mathbf{B}$ (which we take to point along the $z$-direction), are specified in terms of four quantum numbers. In the gauge where $\mathbf{A}(\mathbf{x})=x B \hat{\mathbf{y}}$, these quantum numbers are $p_{z}$ (momentum component along $\mathbf{B}$ ), $p_{y}$ (momentum component along $\mathbf{A}$ ), a non-negative integer $n=0,1,2, \ldots$ that specifies the Landau level, and an integer $\lambda= \pm 1$ which denotes the spin parallel or antiparallel to $\mathbf{B}$. The energy levels are independent of the quantum number $p_{y}$ and are given by [13]

$$
E\left(p_{z}, j\right)=\sqrt{p_{z}^{2}+m^{2}+(2 e B) j},
$$

where $j$ is a nonnegative integer defined by $j=n+(\lambda+1) / 2$. The energy levels (1) are highly degenerate, due to their independence on $p_{y}$ and their dependence on $n$ and $\lambda$ only through the single combination $j$. The number of states $g\left(p_{z}, j\right) d p_{z}$ for given $j$ and momentum along $\mathbf{B}$ between $p_{z}$ and $p_{z}+d p_{z}$, in a system of electrons confined to a finite cubic box of volume $V$, is given by

$$
g\left(p_{z}, j\right) d p_{z}=g_{j} \frac{e B}{4 \pi^{2}} V d p_{z},
$$

with $g_{j}=1$ for $j=0$ and $g_{j}=2$ for $j \neq 0$. Physically, this degeneracy embodies the fact that the levels with $j=0$ accommodate only electrons with spin pointing down, whereas all the others can have electrons spinning in either direction.

\footnotetext{
${ }^{3}$ For $g=2$, i.e., neglecting QED corrections to the electron's magnetic moment.
} 
As an immediate consequence, the levels with $j=0$ alone are responsible for the paramagnetic contribution to the magnetization. See [14] for details. The density of states in energy space is thus given by

$$
g(E) d E=2 \sum_{j=0}^{j_{\mathrm{E}}} g\left(\left|p_{z}\right|, j\right) d p_{z},
$$

where the factor of 2 in front of the sum takes into account both possible signs of $p_{z}$, and the integer $j_{\mathrm{E}}$ corresponds to the highest Landau level $j$ that starts at an energy less than $E$. From Eq. (画) it is clear that $j_{\mathrm{E}}$ is the integer part of the quantity

$$
q_{\mathrm{E}}=\frac{E^{2}-m^{2}}{2 e B},
$$

denoted usually as $j_{\mathrm{E}}=\left\lfloor q_{\mathrm{E}}\right\rfloor$. The degeneracy of the levels [13] can be expressed explicitly by

$$
\begin{aligned}
g(E) & =\frac{V}{4 \pi^{2}} 2 e B E \sum_{j=0}^{j_{\mathrm{E}}} g_{j} \frac{1}{\sqrt{E^{2}-m^{2}-2 e B \cdot j}} \\
& =\frac{V}{4 \pi^{2}}(2 e B)^{1 / 2} E \sum_{j=0}^{\left\lfloor q_{\mathrm{E}}\right\rfloor} g_{j} \frac{1}{\sqrt{q_{\mathrm{E}}-j}} .
\end{aligned}
$$

Considering now the degeneracy $g_{j}$ in Eq. (5) and formula (61) with $z=1 / 2$ we find

$$
\sum_{j=0}^{\left\lfloor q_{\mathrm{E}}\right\rfloor} g_{j} \frac{1}{\sqrt{q_{\mathrm{E}}-j}}=2\left[\zeta\left(\frac{1}{2}, q_{\mathrm{E}}-\left\lfloor q_{\mathrm{E}}\right\rfloor\right)-\zeta\left(\frac{1}{2}, q_{\mathrm{E}}+1\right)-\frac{1}{2 \sqrt{q_{\mathrm{E}}}}\right],
$$

where the r.h.s. of this equation defines the following family of functions that appear regularly in the thermodynamic expressions for this system:

$$
\mathcal{H}_{z}(q) \equiv \zeta(z,\{q\})-\zeta(z, q+1)-\frac{1}{2} q^{-z},
$$

and where $\{q\}=q-\lfloor q\rfloor$ is the fractional part of $q$.

In what follows, we will denote energies in units of the electron mass, $\varepsilon=E / m$, and the magnetic force $e B$ in terms of $m^{2}$ :

$$
b \equiv \frac{2 e B}{m^{2}} .
$$

The variable $b$ measures the magnetic field strength in units of the natural strength $B_{0}=\frac{1}{2} m^{2} c^{4} / e \hbar c \approx 2.2 \times 10^{13}$ Gauss 15$]$.

With this notation and the use of Eq. (9), the density of states for energy $E$ has a simple form:

$$
g(E)=V \frac{m^{2}}{2 \pi^{2}} b^{1 / 2} \varepsilon \mathcal{H}_{1 / 2}\left(\frac{\varepsilon^{2}-1}{b}\right) .
$$


The total number of states up to a given energy $E$ is the integral over the density of states:

$$
G(E)=\int_{m}^{E} g\left(E^{\prime}\right) d E^{\prime}
$$

Using Eq. (62) of Appendix A, it is easy to show that $\mathcal{H}_{1 / 2}(q)=2 \mathcal{H}_{-1 / 2}^{\prime}(q)$ (where the prime denotes differentiation with respect to $q$ ), so that we can do this integral in a closed form, obtaining:

$$
G(E)=V \frac{m^{3}}{2 \pi^{2}} b^{3 / 2} \quad \mathcal{H}_{-1 / 2}\left(\frac{\varepsilon^{2}-1}{b}\right) .
$$

Clearly, $G\left(E_{\mathrm{F}}\right) / V$ is the electron density in the system, where $E_{\mathrm{F}}$ denotes the Fermi energy. We will consistently use dimensionless variables in the expressions:

$$
\varepsilon_{\mathrm{F}} \equiv \frac{E_{\mathrm{F}}}{m}, \quad p_{\mathrm{F}} \equiv \sqrt{\varepsilon_{\mathrm{F}}^{2}-1}
$$

While $\varepsilon_{\mathrm{F}}$ is the Fermi energy, $p_{\mathrm{F}}$ is the Fermi momentum of the $j=0$ Landau level, in units of $m$. The expression $p_{\mathrm{F}}^{2} / b$ gives a measure of the occupation of the Landau levels in the ground state: its integer part, $\left\lfloor p_{\mathrm{F}}^{2} / b\right\rfloor$, equals the last Landau level that is occupied, and its fractional part $\left\{p_{\mathrm{F}}^{2} / b\right\}$, or more precisely $1-\left\{p_{\mathrm{F}}^{2} / b\right\}$, gives a measure of the "distance" to the next empty one.

\section{$3 \quad$ Limiting cases and regimes}

In the non-relativistic case, the energy scale associated with the magnetic field is the spacing between Landau levels, $e B / m$, which is uniform and equal to $\omega_{B}$, the cyclotron frequency. For typical values of magnetic field strengths found in pulsars, say $B=10^{12}$ Gauss, one finds $\omega_{B} \approx 10 \mathrm{keV}$.

As is well known, a fermion gas becomes degenerate when the occupation number falls abruptly for states with energy above a given value $E_{\mathrm{F}}$, the Fermi energy. This happens if the temperature is much smaller than the kinetic range $T_{\mathrm{F}} \equiv E_{\mathrm{F}}-m$ (i.e. the energy range between the lowest orbital and the Fermi energy). For electrons in neutron stars, typical values might be $T<1 \mathrm{MeV}$ and $T_{\mathrm{F}} \simeq 10^{2} \mathrm{MeV}$, so that the gas is not only degenerate but also highly relativistic. In the relativistic regime however, the level spacing is not uniform, but decreases with energy, so that close to the Fermi surface it is $e B / E_{\mathrm{F}}$.

While the Fermi energy is invariably larger than the temperature in a degenerate gas, the number of occupied Landau levels may or may not be large. This number is given by the integer part of $\left(E_{\mathrm{F}}^{2}-m^{2}\right) /(2 e B)$, in view of Eq. (7) and its preceding paragraph, and so it is large if $2 e B / E_{\mathrm{F}} \ll T_{\mathrm{F}}$. This is indeed the 
case for typical magnetic fields in neutron stars $\left(B<10^{14} \mathrm{G}\right)$ where $e B / E_{\mathrm{F}}<10$ $\mathrm{keV}$.

We can still classify the gas in a uniform magnetic field as cold of warm, depending on whether the temperature is smaller or larger than the energy spacing between Landau levels close to the Fermi surface, the latter being $e B / E_{\mathrm{F}}$. Consequently, in a cold degenerate gas, the following condition is satisfied:

$$
T \ll \frac{e B}{E_{\mathrm{F}}} \text { and } T \ll T_{\mathrm{F}} .
$$

If, in addition, the magnetic field is small so that there is a large number of occupied Landau levels, the above hierarchy becomes:

$$
T \ll \frac{e B}{E_{\mathrm{F}}} \ll T_{\mathrm{F}} .
$$

On the other hand, in the warm regime, the temperature is comparable or larger than the Landau spacing:

$$
\frac{e B}{E_{\mathrm{F}}}<T \ll T_{\mathrm{F}} .
$$

These three cases are the regimes of interest here.

\section{The Grand Potential and Hurwitz Functions}

The general expression for the grand potential for an ideal Fermi gas in a uniform background magnetic field $B$ is given by

$$
\Omega(T, B, V, \mu)=-T \sum_{\alpha} \ln \left(1+e^{-\left(E_{\alpha}-\mu\right) / T}\right),
$$

where $\mu$ is the chemical potential and the sum is over all 1-particle orbitals $\alpha$. The $B$ dependence is implicit in the orbital energies $E_{\alpha}$ and the density of states. Thermodynamic quantities can be obtained directly from $\Omega$. In particular, $\mathcal{M}$, the magnetization per unit volume is obtained as

$$
\mathcal{M}=-\frac{1}{V} \frac{\partial \Omega}{\partial B} .
$$

In terms of the density of states $g(E)$ the grand potential (per unit volume) is

$$
\omega \equiv \frac{\Omega}{V}=-T \int_{m}^{\infty} \frac{g(E)}{V} \ln \left(1+e^{-(E-\mu) / T}\right) d E .
$$

This expression, together with the explicit form (12) for $g(E)$, can be used to study the thermodynamics at arbitrary temperature $T$ and magnetic field $B$. 


\section{The $T=0$ Limit}

As we show in Appendix B, for a system of non-interacting fermions any finite temperature quantity can be obtained from its zero-temperature counterpart. Therefore, in the rest of this section we will just concentrate on the grand potential density at zero temperature, $\omega(T=0) \equiv \omega_{0}$, keeping in mind the standard definition for the Fermi energy $E_{\mathrm{F}} \equiv \mu(T=0)$. The $T=0$ limit is simply formulated from

$$
\lim _{T \rightarrow 0}-T \ln \left(1+e^{-(E-\mu) / T}\right)= \begin{cases}E-E_{\mathrm{F}} & \text { for } E<E_{\mathrm{F}} \\ 0 & \text { for } E>E_{\mathrm{F}}\end{cases}
$$

so that

$$
\omega_{0}\left(E_{\mathrm{F}}, B\right)=\int_{m}^{E_{\mathrm{F}}} \frac{g(E)}{V} E d E-E_{\mathrm{F}} \frac{N}{V} .
$$

The integral in Eq. (23), which is the ground state energy density $\left(\equiv u_{0}\right)$, can be done using the explicit representation for the density of states given in Eq. (12). After standard manipulation and integration by parts we get:

$$
\begin{aligned}
u_{0} \equiv \int_{m}^{E_{\mathrm{F}}} \frac{g(E)}{V} E d E=\frac{m^{4}}{4 \pi^{2}} & {\left[2 b^{3 / 2} \varepsilon_{\mathrm{F}} \mathcal{H}_{-1 / 2}\left(\frac{p_{\mathrm{F}}^{2}}{b}\right)\right.} \\
& \left.-b^{5 / 2} \int_{0}^{p_{\mathrm{F}}^{2} / b} \frac{\mathcal{H}_{-1 / 2}(q)}{\sqrt{1+b q}} d q\right] .
\end{aligned}
$$

Notice that the first term above is exactly $E_{\mathrm{F}} N / V$, according to Eq. (14), so it

cancels with the second term in Eq. (23), leaving a compact form for the grand potential density $\omega_{0} \equiv \Omega_{0} / V$ :

$$
\omega_{0}\left(\varepsilon_{\mathrm{F}}, b\right)=-\frac{m^{4}}{4 \pi^{2}} b^{5 / 2} \int_{0}^{p_{\mathrm{F}}^{2} / b} \frac{\mathcal{H}_{-1 / 2}(q)}{\sqrt{1+b q}} d q .
$$

where

$$
\mathcal{H}_{-1 / 2}(q)=\zeta\left(-\frac{1}{2},\{q\}\right)-\zeta\left(-\frac{1}{2}, q+1\right)-\frac{1}{2} \sqrt{q} .
$$

Formally, this is the main result of this work.

Expression (25) allows us to recover the well known non-relativistic result 12 in a straightforward fashion: in the non-relativistic limit $p_{\mathrm{F}} \ll 1$, so that the square root in the integrand of (25) can simply be replaced by unity. Using then the relation $\mathcal{H}_{-1 / 2}(q)=\frac{2}{3} \mathcal{H}_{-3 / 2}^{\prime}(q)$ we find

$$
\omega_{0}^{(\text {n.r. })}\left(\varepsilon_{\mathrm{F}}, b\right)=-\frac{m^{4}}{6 \pi^{2}} b^{5 / 2} \mathcal{H}_{-3 / 2}\left(p_{\mathrm{F}}^{2} / b\right),
$$

where

$$
\mathcal{H}_{-3 / 2}(q) \equiv \zeta\left(-\frac{3}{2},\{q\}\right)-\zeta\left(-\frac{3}{2}, q+1\right)-\frac{1}{2} q^{3 / 2} .
$$


The result (27) is exactly the known non-relativistic result, after identifying $p_{\mathrm{F}}^{2}=2 \mu_{n . r .} / m$, where $\mu_{n . r .}$ is the standard (non-relativistic) chemical potential. In particular, notice that $\mathcal{H}_{-3 / 2}(q)$ splits naturally into a monotonic term and an oscillatory term, $\mathcal{H}_{-3 / 2}(q)=\mathcal{H}_{-3 / 2}^{(\mathrm{mon})}(q)+\mathcal{H}_{-3 / 2}^{\text {(osc) }}(q)$, as:

$$
\begin{aligned}
\mathcal{H}_{-3 / 2}^{(\mathrm{mon})}(q) & =-\zeta\left(-\frac{3}{2}, q+1\right)-\frac{1}{2} q^{3 / 2} \\
\mathcal{H}_{-3 / 2}^{(\mathrm{osc})}(q) & =\zeta\left(-\frac{3}{2},\{q\}\right) .
\end{aligned}
$$

The oscillating character of $\mathcal{H}_{-3 / 2}^{(\mathrm{osc})}(q)$ arises from the fractional part $\{q\}$ in the definition above. This is the term that accounts for the de Haas - van Alphen oscillations in the non-relativistic case, and leads to Landau's result at finite temperature, as it is shown in section 5 .

Likewise, the relativistic grand potential density (25) separates into two terms, $\omega_{0}=\omega_{0}^{(\mathrm{mon})}+\omega_{0}^{(\mathrm{osc})}$, where

$$
\begin{aligned}
\omega_{0}^{(\mathrm{mon})}\left(\varepsilon_{\mathrm{F}}, b\right) & =\frac{m^{4}}{4 \pi^{2}} b^{5 / 2} \int_{0}^{p_{\mathrm{F}}^{2} / b} \frac{\zeta\left(-\frac{1}{2}, q+1\right)+\frac{1}{2} \sqrt{q}}{\sqrt{1+b q}} d q, \\
\omega_{0}^{(\mathrm{osc})}\left(\varepsilon_{\mathrm{F}}, b\right) & =-\frac{m^{4}}{4 \pi^{2}} b^{5 / 2} \int_{0}^{p_{\mathrm{F}}^{2} / b} \frac{\zeta\left(-\frac{1}{2},\{q\}\right)}{\sqrt{1+b q}} d q .
\end{aligned}
$$

Here we have kept the notation consistent, however one must be aware that $\omega_{0}^{\text {(osc) }}$, the integral of an oscillating function, is not purely oscillatory, as we will see. The integral expressions above are exact and finite for any values of the magnetic field and Fermi momentum.

Although the integrals in Eqs. (31) and (32) cannot be solved in a closed form, one can nevertheless use the analytic properties of the Hurwitz zeta function to obtain an expansion of $\omega_{0}$ for small $b$, or similarly for large $p_{\mathrm{F}}^{2} / b$ (i.e. large number of occupied Landau levels). Let us first consider $\omega_{0}^{(\mathrm{mon})}$. A naïve binomial expansion of the square root in the integrand will not work because of the diverging behavior of the integral as $p_{\mathrm{F}}^{2} / b \rightarrow \infty$. A procedure to obtain the correct expansion for small $b$ consists in first extracting out a sufficient number of leading terms in the asymptotic expansion of the zeta function for large $q$, such that the remainder is integrable in the limit $b \rightarrow 0$ [three terms will do for $\zeta(-1 / 2, q)$ ], and then integrating the subtracted terms explicitly. Based on Eq. (71) of Appendix A we write

$$
\zeta(z, q)=\frac{1}{z-1} q^{-z+1}+\frac{1}{2} q^{-z}+\frac{z}{12} q^{-z-1}+\tilde{\zeta}_{3}(z, q),
$$

implicitly defining the subtracted zeta function $\tilde{\zeta}_{3}(z, q)$. Now, the integrals corresponding to the exhibited terms in (33) along with the term $\sqrt{q} / 2$ in (31) can be done exactly for finite $b$, leaving only an integral containing the subtracted 
function $\tilde{\zeta}_{3}(-1 / 2, q)$ :

$$
\begin{aligned}
\omega_{0}^{(\text {mon })}\left(\varepsilon_{\mathrm{F}}, b\right)=-\frac{m^{4}}{4 \pi^{2}} & \left\{\frac{1}{2}\left(1-b+\frac{1}{6} b^{2}\right) \ln \left(\frac{\varepsilon_{\mathrm{F}}+\sqrt{b+p_{\mathrm{F}}^{2}}}{1+\sqrt{b}}\right)\right. \\
& -\frac{1}{2}\left[\left(b+p_{\mathrm{F}}^{2}\right)^{1 / 2} \varepsilon_{\mathrm{F}}-b^{1 / 2}\right] \\
& +\frac{1}{3}\left[\left(b+p_{\mathrm{F}}^{2}\right)^{3 / 2} \varepsilon_{\mathrm{F}}-b^{3 / 2}\right] \\
& +\frac{1}{2} b\left[\cosh ^{-1}\left(\varepsilon_{\mathrm{F}}\right)-p_{\mathrm{F}} \varepsilon_{\mathrm{F}}\right] \\
& \left.-b^{5 / 2} \int_{0}^{p_{\mathrm{F}}^{2} / b} \frac{\tilde{\zeta}_{3}\left(-\frac{1}{2}, q+1\right)}{\sqrt{1+b q}} d q\right\} .
\end{aligned}
$$

The expression above is still exact, but now the integral containing $\tilde{\zeta}_{3}(z, q)$ can be expanded in powers up to order $b$ without entering into trouble, because $\tilde{\zeta}_{3}(-1 / 2, q)$ vanishes as $q^{-5 / 2}$ for $q \rightarrow \infty$ (see Eq. (73) of Appendix A). It suffices to take the upper limit to infinity and expand the denominator:

$$
\begin{aligned}
\int_{0}^{p_{\mathrm{F}}^{2} / b} \frac{\tilde{\zeta}_{3}\left(-\frac{1}{2}, q+1\right)}{\sqrt{1+b q}} d q \stackrel{b \rightarrow 0}{\longrightarrow} & -\left(\frac{2}{3} \zeta\left(-\frac{3}{2}\right)+\frac{1}{60}\right) \\
& +b\left(-\frac{2}{15} \zeta\left(-\frac{5}{2}\right)+\frac{1}{1260}\right)+O\left(b^{3 / 2}\right) \ldots
\end{aligned}
$$

This integral thus loses the dependence on the chemical potential and becomes a spurious contribution that cancels in $\omega_{0}$ when we put all pieces together (see below). A word of caution is due: to go to higher orders in $b$ one cannot continue this expansion in the same fashion. Instead, one should follow the same procedure from the beginning, but extracting more explicit terms from the asymptotic expansion (33), integrating them directly, and then expanding the $n$-residual integral containing $\tilde{\zeta}_{n}(-1 / 2, q)$ up to the last convergent term, that goes as $b^{2 n-5}$.

Now, let us turn to the oscillatory contribution $\omega_{0}^{\text {(osc) }}$, which can be written in the following form (see Appendix C):

$$
\begin{aligned}
\omega_{0}^{(\mathrm{osc})}= & -\frac{m^{4}}{4 \pi^{2}} b^{2}\left\{\int_{0}^{\left\{p_{\mathrm{F}}^{2} / b\right\}} \frac{\zeta\left(-\frac{1}{2}, q\right)}{\sqrt{q+1 / b+\left\lfloor p_{\mathrm{F}}^{2} / b\right\rfloor}} d q\right. \\
& \left.+\int_{0}^{1} \zeta\left(-\frac{1}{2}, q\right)\left[\zeta\left(\frac{1}{2}, q+1 / b\right)-\zeta\left(\frac{1}{2}, q+1 / b+\left\lfloor p_{\mathrm{F}}^{2} / b\right\rfloor\right)\right] d q\right\}
\end{aligned}
$$

whose expansion for small $b$ is straightforward (however tedious):

$$
\omega_{0}^{(\mathrm{osc})} \stackrel{b \rightarrow 0}{\longrightarrow}-\frac{m^{4}}{4 \pi^{2}}\left\{\frac{2}{3} b^{5 / 2}\left[\frac{1}{\varepsilon_{\mathrm{F}}} \zeta\left(-\frac{3}{2},\left\{p_{\mathrm{F}}^{2} / b\right\}\right)-\zeta\left(-\frac{3}{2}\right)\right]\right.
$$




$$
\left.+\frac{2}{15} b^{7 / 2}\left[\frac{1}{\varepsilon_{\mathrm{F}}^{3}} \zeta\left(-\frac{5}{2},\left\{p_{\mathrm{F}}^{2} / b\right\}\right)-\zeta\left(-\frac{5}{2}\right)\right]\right\}+O\left(b^{9 / 2}\right) \ldots
$$

Finally we can put all the terms together and find the expansion of the grand potential density $\omega_{0}$ for small magnetic field (see Appendix D):

$$
\begin{aligned}
\omega_{0}=\quad & -\frac{m^{4}}{4 \pi^{2}}\left\{\frac{1}{2} \cosh ^{-1}\left(\varepsilon_{\mathrm{F}}\right)+\frac{1}{3} \varepsilon_{\mathrm{F}} p_{\mathrm{F}}^{3}-\frac{1}{2} \varepsilon_{\mathrm{F}} p_{\mathrm{F}}\right. \\
& +\frac{b^{2}}{12} \cosh ^{-1}\left(\varepsilon_{\mathrm{F}}\right)+\frac{2}{3} \frac{b^{5 / 2}}{\varepsilon_{\mathrm{F}}} \zeta\left(-\frac{3}{2},\left\{p_{\mathrm{F}}^{2} / b\right\}\right) \\
& \left.+\frac{2}{15} \frac{b^{7 / 2}}{\varepsilon_{\mathrm{F}}^{3}} \zeta\left(-\frac{5}{2},\left\{p_{\mathrm{F}}^{2} / b\right\}\right)+O\left(b^{4}\right) \ldots\right\} .
\end{aligned}
$$

We note that expansion (38) is in agreement, apart from an overall sign, with the small field result for the effective action obtained in Ref. [9] (Eq. (74)), which in our notation reads

$$
\begin{aligned}
& \left.\Delta S^{d=3}\right|_{T=0 ; \mu \neq 0}=\frac{b m^{4}}{8 \pi^{2}}\left\{\varepsilon_{\mathrm{F}} p_{\mathrm{F}}-\ln \left(\varepsilon_{\mathrm{F}}+p_{\mathrm{F}}\right)\right\} \\
& +\frac{b^{2} m^{4}}{2 \pi^{2}} \sum_{l=0}^{\infty} \frac{\left(b / \varepsilon_{\mathrm{F}}^{2}\right)^{l+1 / 2}}{(2 l+1)(2 l+3)}\left\{\zeta\left(-l-\frac{3}{2},\left\{p_{\mathrm{F}}^{2} / b\right\}\right)-\zeta\left(-l-\frac{3}{2}, p_{\mathrm{F}}^{2} / b\right)\right\}
\end{aligned}
$$

Although the non-analytic oscillatory terms are immediate to compare, it is quite nontrivial to obtain the analytic terms from (39), since each term of the type $b^{l+1 / 2} \zeta\left(-l-\frac{3}{2}, p_{\mathrm{F}}^{2} / b\right)$ in the sum in (39) contributes to all orders in $b$ as $b \rightarrow 0$, in view of the asymptotic expansion (72) for the Hurwitz zeta function. Using the first three terms of this asymptotic expansion, one can perform each of the resulting infinite sums in a closed way to indeed obtain the terms shown in (38), with a vanishing coefficient for the term linear in $b$. In retrospect, we realize that the result $(39)$ can be formally obtained directly from the closed expression (25) by doing a binomial expansion of the square root and then integrating term by term. However, the latter expansion is valid over the whole integration range only if $p_{\mathrm{F}}^{2}<1$, i.e. basically in the non-relativistic limit. From a numerical point of view it is clearly advantageous to use expansion (38) instead of (39), since then one can compute to any desired precision at small $b$ by keeping only a finite number of terms in the expansion.

All thermodynamic quantities at $T=0$ can be obtained from $\omega_{0}$ as derivatives. One should notice that, since $\zeta(z, 0)=\zeta(z, 1)$ for $z<0$, the function $\zeta(z,\{q\})$ is continuous in $q$ for all $z<0$ and, in view of Eq. (62), has continuous derivative for all $z<-1$. For example, it is straightforward to derive the expansion for the density at $T=0$ using the thermodynamic identity

$$
n_{0}=-\frac{1}{m} \frac{\partial \omega_{0}}{\partial \varepsilon_{\mathrm{F}}} .
$$


We thus find the the density at $T=0$, consistent with Eq. (14), and the corresponding expansion for small $b$ :

$$
\begin{aligned}
n_{0}\left(\varepsilon_{\mathrm{F}}, b\right) & =\frac{m^{3}}{2 \pi^{2}} b^{3 / 2} \mathcal{H}_{-1 / 2}\left(\frac{p_{\mathrm{F}}^{2}}{b}\right) \\
& \rightarrow \frac{m^{3}}{2 \pi^{2}}\left\{\frac{2}{3} p_{\mathrm{F}}^{3}+b^{3 / 2} \zeta\left(-\frac{1}{2},\left\{p_{\mathrm{F}}^{2} / b\right\}\right)+\frac{b^{2}}{24 p_{\mathrm{F}}}+O\left(b^{4}\right) \ldots\right\}
\end{aligned}
$$

In the $b=0$ limit we recover the free electron gas result, $n_{0}=m^{3} p_{\mathrm{F}}^{3} / 3 \pi^{2}$. As seen in Eq. (41), the leading correction for finite $b$ and $T=0$ is a term that oscillates with $p_{\mathrm{F}}^{2} / b$, corresponding to the de Haas-van Alphen effect for the magnetization in metals.

Fig. 1 shows $n_{0}$ vs. $\varepsilon_{\mathrm{F}}$ for two values of the field. The step-like behavior is due to the filling of consecutive Landau levels as $\varepsilon_{\mathrm{F}}$ increases. The density of states in a given Landau level [see Eq. (5)] goes as $b d p_{z} \sim b d E / p_{z}$, for $p_{z}$ starting from zero. The higher density at the bottom of each level causes the step in $n_{0}$. On the other hand, as $b$ decreases the steps gradually disappear until the smooth $b=0$ limit is reached. Alternatively, Fig. 2 shows $n_{0}$ as a function of the field $b$, for fixed Fermi energy. Here $n_{0}$ oscillates as $b$ changes. Imagine we start from a large value of $b$. As $b$ decreases, the Landau levels move down in energy, crossing one by one the threshold defined by $\varepsilon_{\mathrm{F}}$. Since the levels are denser at the bottom, $n_{0}$ first grows as each level becomes accessible, but then decreases again, because of the $b$ factor in the density of states. The oscillation amplitude becomes smaller and smaller as the Landau levels get closer and closer, until we reach the $b=0$ limit.

We note that the expansion in Eq. (41) is actually a large $p_{\mathrm{F}}^{2} / b$ expansion, appropriate when a large number of Landau levels are occupied at $T=0$. So it will remain valid even for large values of the magnetic field, say $b \sim 100$, provided the ratio $p_{\mathrm{F}}^{2} / b$ stays much larger than one. This will be the case for electron densities in typical neutron stars, for which $p_{\mathrm{F}}^{2} \sim 10^{4}$.

We can also obtain the expansion for $u_{0}$, the energy density at $T=0$-whose exact integral form is given in Eq. (24) - , using the expansions in Eqs. (38) and (41) and the thermodynamic relation $u_{0}=\omega_{0}+m \varepsilon_{\mathrm{F}} n_{0}$ :

$$
\begin{aligned}
u_{0}= & \frac{m^{4}}{4 \pi^{2}}\left\{\varepsilon_{\mathrm{F}} p_{\mathrm{F}}^{3}+\frac{1}{2} \varepsilon_{\mathrm{F}} p_{\mathrm{F}}-\frac{1}{2} \cosh ^{-1}\left(\varepsilon_{\mathrm{F}}\right)\right. \\
& +2 b^{3 / 2} \varepsilon_{\mathrm{F}} \zeta\left(-\frac{1}{2},\left\{p_{\mathrm{F}}^{2} / b\right\}\right)+\frac{b^{2}}{12} \frac{\varepsilon_{\mathrm{F}}}{p_{\mathrm{F}}^{2}}-\frac{b^{2}}{12} \cosh ^{-1}\left(\varepsilon_{\mathrm{F}}\right) \\
& -\frac{2}{3} \frac{b^{5 / 2}}{\varepsilon_{\mathrm{F}}} \zeta\left(-\frac{3}{2},\left\{p_{\mathrm{F}}^{2} / b\right\}\right)-\frac{2}{15} \frac{b^{7 / 2}}{\varepsilon_{\mathrm{F}}^{3}} \zeta\left(-\frac{5}{2},\left\{p_{\mathrm{F}}^{2} / b\right\}\right) \\
& \left.+O\left(b^{4}\right) \ldots\right\} .
\end{aligned}
$$


Unlike Eq. (41) for the particle density, the expansions for the energy density given above and for the grand potential given in Eq. (38) are valid for small $b$ only. More useful in the case of neutron star conditions is an expansion for large $p_{\mathrm{F}}^{2} / b$, that is, many Landau levels occupied, but regardless of $b$. In that case, for instance, the small $b$ expansion of the denominator in Eq. (35) is not valid. The correct expansion of $\omega_{0}$ for large $p_{\mathrm{F}}^{2} / b$ is the following, where some $p_{\mathrm{F}}$-independent integrals are left to be done numerically:

$$
\begin{aligned}
& \omega_{0}^{(\mathrm{mon})}\left(\varepsilon_{\mathrm{F}}, b\right)=-\frac{m^{4}}{4 \pi^{2}}\left\{\frac{1}{2}\left(1-b+\frac{1}{6} b^{2}\right) \ln \left(\frac{\varepsilon_{\mathrm{F}}+\sqrt{b+p_{\mathrm{F}}^{2}}}{1+\sqrt{b}}\right)\right. \\
& -\frac{1}{2}\left[\left(b+p_{\mathrm{F}}^{2}\right)^{1 / 2} \varepsilon_{\mathrm{F}}-b^{1 / 2}\right] \\
& +\frac{1}{3}\left[\left(b+p_{\mathrm{F}}^{2}\right)^{3 / 2} \varepsilon_{\mathrm{F}}-b^{3 / 2}\right] \\
& +\frac{1}{2} b\left[\cosh ^{-1}\left(\varepsilon_{\mathrm{F}}\right)-p_{\mathrm{F}} \varepsilon_{\mathrm{F}}\right] \\
& \left.-b^{5 / 2} \int_{0}^{\infty} \frac{\tilde{\zeta}_{3}\left(-\frac{1}{2}, q+1\right)}{\sqrt{1+b q}} d q+\frac{1}{3840} \frac{b^{4}}{\varepsilon_{\mathrm{F}}^{4}}+\ldots\right\}, \\
& \omega_{0}^{(\mathrm{osc})}=-\frac{m^{4}}{4 \pi^{2}}\left\{\frac{2}{3} \frac{b^{5 / 2}}{\varepsilon_{\mathrm{F}}} \zeta\left(-\frac{3}{2},\left\{p_{\mathrm{F}}^{2} / b\right\}\right)+\frac{2}{15} \frac{b^{7 / 2}}{\varepsilon_{\mathrm{F}}^{3}} \zeta\left(-\frac{5}{2},\left\{p_{\mathrm{F}}^{2} / b\right\}\right)+\ldots\right. \\
& \left.+b^{2} \int_{0}^{1} \zeta\left(-\frac{1}{2}, q\right) \zeta\left(\frac{1}{2}, q+1 / b\right) d q\right\} \text {. }
\end{aligned}
$$

\section{The finite temperature case}

As shown in Appendix B, the grand potential at finite temperature can be obtained from its expression at $T=0, \omega_{0}\left(\varepsilon_{\mathrm{F}}\right)$, as:

$$
\omega(T, \mu)=\int_{-\frac{\mu-1}{T}}^{\infty} \omega_{0}(\mu+T x) \frac{e^{x}}{\left(e^{x}+1\right)^{2}} d x,
$$

where the temperature $T$ and the chemical potential $\mu$ are given in units of the electron mass. If $\omega_{0}(\mu+T x)$ is a slowly varying function of $x$ over a range $\Delta x \sim 1$, then the integral in Eq. (45) can be easily expanded in powers of $T$ :

$$
\omega(T, \mu)=\omega_{0}(\mu)+\frac{\pi^{2}}{6} T^{2} \omega_{0}^{\prime \prime}(\mu)+\frac{7 \pi^{4}}{360} T^{4} \omega_{0}^{(4)}(\mu)+\ldots
$$

In our case of interest, this approximation is valid for the non-oscillatory terms $\omega_{0}^{(m o n)}$, but not for the oscillatory terms, which vary considerably over the range $\Delta x \sim 1$ (unless the unlikely condition $\mu T / b \ll 1$ is met).

The treatment of the oscillatory terms, which are of the form $\zeta\left(z,\left\{\left(\mu^{2}-1\right) / b\right\}\right)$, for $z$ a negative semi-integer, can be done as follows. Consider placing the 
leading oscillatory term,

$$
\omega_{0}^{(\mathrm{osc})} \approx-\frac{m^{4}}{4 \pi^{2}}\left\{\frac{2}{3} \frac{b^{5 / 2}}{\varepsilon_{\mathrm{F}}} \zeta\left(-\frac{3}{2},\left\{p_{\mathrm{F}}^{2} / b\right\}\right)+\ldots\right\},
$$

into the integral of Eq. 445). Accordingly, we must evaluate this function at $\varepsilon_{\mathrm{F}}^{\prime}=$ $\mu+T x$ and $p_{\mathrm{F}}^{\prime 2} \equiv \varepsilon_{\mathrm{F}}^{\prime 2}-1=p_{\mathrm{F}}^{2}+2 \mu T x+T^{2} x^{2}$ (where $T$ is the temperature in units of the electron mass) and weight it with the hump function $h(x)=e^{x} /\left(e^{x}+1\right)^{2}$. In the degenerate regime, $T \ll \mu$, so the term $\mathcal{O}\left(T^{2}\right)$ in the expression for ${p_{\mathrm{F}}^{\prime}}^{2}$ can be safely neglected. Since the prefactor of the zeta function in (47) is a slowly varying function of $x$, we can approximate

$$
\omega^{(\mathrm{osc})}(T, \mu, b) \simeq-\frac{m^{4}}{6 \pi^{2}} \frac{b^{5 / 2}}{\mu} \int_{-\infty}^{\infty} \zeta\left(-\frac{3}{2},\left\{p_{\mathrm{F}}^{2} / b+2 x \mu T / b\right\}\right) h(x) d x .
$$

The number of oscillations that fall under the hump will clearly be proportional to the factor $2 \mu T / b$. We expect the amplitude of the oscillatory magnetization to be more or less constant for $\mu T / b<1$ and rapidly decreasing for $\mu T / b>1$. To obtain an explicit expression we use Hurwitz's Fourier expansion of $\zeta(z, q)$ shown in Eq. (76) (valid for $z<0$ and $0 \leq q \leq 1$ ) and integrate term by term using the formula:

$$
\int_{-\infty}^{\infty} e^{i a x} h(x) d x=\frac{\pi a}{\sinh (\pi a)}
$$

Defining the integral in Eq. (48) in a generic form as:

$$
I_{z}(\alpha, \beta)=\int_{-\infty}^{\infty} \zeta(z,\{\alpha+\beta x\}) h(x) d x,
$$

we find it to be

$$
I_{z}(\alpha, \beta)=(2 \pi)^{z+1} \Gamma(1-z) \beta \sum_{n=1}^{\infty} \frac{n^{z} \sin (2 \pi n \alpha+z \pi / 2)}{\sinh \left(2 \pi^{2} \beta n\right)} .
$$

Now, using this result for $z=-3 / 2$, the oscillatory part of the grand potential becomes:

$$
\omega^{(\mathrm{osc})}(T, \mu, b) \simeq+\frac{m^{4}}{4 \pi^{2}}\left\{b^{3 / 2} \frac{T}{\sqrt{2}} \sum_{n=1}^{\infty} \frac{\cos \left(2 \pi n p_{\mathrm{F}}^{2} / b-\pi / 4\right)}{n^{3 / 2} \sinh \left(4 \pi^{2} n \mu T / b\right)}+\ldots\right\} .
$$

\section{The Magnetization}

The $T=0$ limit 
The magnetization (per unit volume) of the electron gas at $T=0$ is given by

$$
\begin{aligned}
\mathcal{M}_{0}=-\frac{\partial \omega_{0}\left(\varepsilon_{\mathrm{F}}, B\right)}{\partial B} & =-\frac{2 e}{m^{2}} \times \frac{\partial \omega_{0}\left(\varepsilon_{\mathrm{F}}, b\right)}{\partial b} \\
& =\frac{e m^{2}}{2 \pi^{2}} \frac{\partial}{\partial b}\left[b^{5 / 2} \int_{0}^{p_{\mathrm{F}}^{2} / b} \frac{\mathcal{H}_{-1 / 2}(q)}{\sqrt{1+b q}} d q\right] .
\end{aligned}
$$

Taking the derivative we find the formal expression for the magnetization:

$$
\begin{aligned}
\mathcal{M}_{0}\left(\varepsilon_{\mathrm{F}}, b\right)= & \frac{e m^{2}}{2 \pi^{2}}\left\{b^{3 / 2} \int_{0}^{p_{\mathrm{F}}^{2} / b} \mathcal{H}_{-1 / 2}(q)\left[\frac{2}{(1+b q)^{1 / 2}}+\frac{1}{2(1+b q)^{3 / 2}}\right] d q\right. \\
& \left.-b^{1 / 2} \frac{p_{\mathrm{F}}^{2}}{\varepsilon_{\mathrm{F}}} \mathcal{H}_{-1 / 2}\left(p_{\mathrm{F}}^{2} / b\right)\right\} .
\end{aligned}
$$

Just as $\omega_{0}$ was separated into two terms according to the monotonic and the oscillating parts of $\mathcal{H}_{-1 / 2}$, so can we separate the magnetization as $\mathcal{M}_{0}\left(\varepsilon_{\mathrm{F}}, b\right)=$ $\mathcal{M}_{0}^{\text {(mon) }}\left(\varepsilon_{\mathrm{F}}, b\right)+\mathcal{M}_{0}^{\text {(osc) }}\left(\varepsilon_{\mathrm{F}}, b\right)$. The small $b$ expansion for $\mathcal{M}_{0}$ can be obtained from Eq. (54), or directly taking the derivative of the expansion for $\omega_{0}$ given in Eq. (38):

$$
\begin{aligned}
\mathcal{M}_{0}\left(\varepsilon_{\mathrm{F}}, b\right) & =\frac{e m^{2}}{2 \pi^{2}}\left\{-b^{1 / 2} \frac{p_{\mathrm{F}}^{2}}{\varepsilon_{\mathrm{F}}} \zeta\left(-\frac{1}{2},\left\{p_{\mathrm{F}}^{2} / b\right\}+\frac{1}{6} b \cosh ^{-1}\left(\varepsilon_{\mathrm{F}}\right)\right)\right. \\
& +\frac{b^{3 / 2}}{3} \frac{\left(4 \varepsilon_{\mathrm{F}}^{2}+1\right)}{\varepsilon_{\mathrm{F}}^{3}} \zeta\left(-\frac{3}{2},\left\{p_{\mathrm{F}}^{2} / b\right\}\right)+\frac{b^{5 / 2}}{15} \frac{\left(4 \varepsilon_{\mathrm{F}}^{2}+3\right)}{\varepsilon_{\mathrm{F}}^{5}} \zeta\left(-\frac{5}{2},\left\{p_{\mathrm{F}}^{2} / b\right\}\right) \\
& \left.+O\left(b^{3}\right) \ldots\right\} .
\end{aligned}
$$

The magnetization oscillates as a function of $b$ (see Fig. 3), just like the well known de Haas-van Alphen effect of non-relativistic electrons in metals. In this expression, the oscillation appears in terms of Hurwitz functions of the fractional part of $p_{\mathrm{F}}^{2} / b$. Notice that, for $b \lesssim 1$, the oscillatory part has an amplitude considerably larger than the monotonic part, and even larger the larger $\varepsilon_{\mathrm{F}}$ is (see envelope curves in Fig. 3). As such, it could be possible to have spontaneous magnetization for sufficiently dense systems at low temperature. A discussion about the thermodynamic stability of such state was given in Ref. [2]. However, at temperatures above some threshold, the oscillation amplitude dies out, and with it the possibility of spontaneous magnetization [3, [].

\section{The finite temperature case}

We will be concerned in particular with the physically relevant case where $p_{\mathrm{F}}^{2} / b \gg 1$, i.e. many Landau levels occupied. In this case, the dominant contribution at $T=0$ is the leading oscillatory term followed by the leading monotonic term, i.e. the second and first terms in Eq. (55), respectively. 
At finite temperature, the monotonic term becomes:

$$
\begin{aligned}
\mathcal{M}^{(\mathrm{mon})}(T, \mu, b) \simeq & \frac{e m^{2}}{2 \pi^{2}} \frac{b}{6}\left\{\cosh ^{-1}(\mu)\right. \\
& \left.-\frac{\pi^{2}}{6} \frac{\mu}{p_{\mathrm{F}}^{3}} T^{2}-\frac{7 \pi^{4}}{60} \frac{\mu\left(\mu^{2}+\frac{3}{2}\right)}{p_{\mathrm{F}}^{7}} T^{4}+\ldots\right\},
\end{aligned}
$$

where $p_{\mathrm{F}}^{2} \equiv \mu^{2}-1$. This contribution is small compared to the oscillatory part for temperatures smaller than the Landau level splittings, i.e. $T<b / \mu$. However, for higher $T$ the amplitude of the oscillations gets smoothed out and it is the monotonic part shown above what dominates the magnetization. This behavior is shown in Fig. 4.

On the other hand, the leading oscillatory term at finite $T$ is obtained following the same procedure as in the previous section, or directly taking the derivative of the grand potential with respect to $b$ :

$$
\mathcal{M}^{\text {(osc })}(T, \mu, b)=-\frac{e m^{2}}{\pi} \frac{p_{\mathrm{F}}^{2} T}{\sqrt{2 b}} \sum_{n=1}^{\infty} \frac{\sin \left(2 \pi n p_{\mathrm{F}}^{2} / b-\pi / 4\right)}{\sqrt{n} \sinh \left(4 \pi^{2} n \mu T / b\right)}+\ldots
$$

This expression coincides with the results in Ref. 渚. We must also remark the strong similitude with Landau's result for the non-relativistic case [12], which is expressed in our notation as:

$$
\mathcal{M}_{n . r .}^{\text {(osc) }}(T, \mu, b)=-\frac{e m^{2}}{\pi} \frac{p_{\mathrm{F}}^{2} T}{\sqrt{2 b}} \sum_{n=1}^{\infty} \frac{\sin \left(2 \pi n p_{\mathrm{F}}^{2} / b-\pi / 4\right)}{\sqrt{n} \sinh \left(4 \pi^{2} n T / b\right)} .
$$

This is precisely the non-relativistic limit of our leading oscillating term, shown in Eq. (57): basically, the relativistic chemical potential reduces to $\mu \equiv 1+$ $\mu_{n . r .} \rightarrow 1$, in units of electron mass, while $p_{\mathrm{F}} \equiv \sqrt{\mu^{2}-1} \rightarrow \sqrt{2 \mu_{n . r .}}$ denotes a non-trivial quantity in all regimes.

A final important point is the possibility of ferromagnetic behavior, that is, a magnetization that is sustained with the magnetic field generated by the same system. For a given geometry, the field and magnetization satisfy a relation of the form $M=\gamma B$, where in the case of $e . g$. a sphere, $\gamma=3 / 8 \pi$. A self-consistent solution is obtained from this relation and the thermodynamic relation $M=$ $M(T, \mu, B)$. This problem has been studied in the past, and our conclusions agree with those results. For the self-consistent solution to exist, the oscillations are necessary, because the monotonic part of $M$ vs. $b$ is simply too small to reach a solution with $M=\gamma B$. Moreover, for the oscillations not to be thermally damped out, the temperature must be below some threshold value [3]. Finally, there is the question of thermodynamic stability of the self-consistent solution, which is at most metastable according to O'Connell and Roussel [2].

\section{Conclusions}


We have developed a closed analytical approach to solve the thermodynamics of a free gas of electrons immersed in an uniform magnetic field of arbitrary magnitude. The method is completely relativistic and particularly useful in the case of a degenerate gas, a likely situation to be met in the interior of white dwarfs, neutron stars, and magnetars. A central role is played by one of the least known of the special functions of mathematical physics, the Hurwitz zeta function. Its appearance comes about due to a sum over energy levels of the form $(\alpha+\beta n)^{z}$ (Landau levels for the system treated in this paper), labeled by a non-negative integer $n$. As such, the method should be applicable to study the thermodynamics of other systems. The grand potential (and therefore all thermodynamic quantities) can be expressed as a one-dimensional definite integral, which can be explicitly evaluated in several interesting regimes due to the various analytic properties of the Hurwitz zeta function. Hence, our work provides a unified derivation of several of the results found scattered in the

literature. We reproduce the de Haas-van Alphen behavior of the magnetization in the relativistic gas and the dilution of it at high temperatures.

We have not included quantum electrodynamics corrections in this treatment. Work to extend our results in this direction, in a fully relativistic fashion, is in progress.

\section{Acknowledgments}

This work was supported by CONICYT under Grant Fondecyt PLC-8000017. 


\section{Appendix A: The Hurwitz Zeta Function}

We collect here some of the properties of the Hurwitz zeta function and present a (presumably original) derivation of its asymptotic behavior for large $q$. For a detailed account consult, for instance, references [16] or [17].

The Hurwitz zeta function $\zeta(z, q)$ is defined as the analytic extension to the whole complex $z$ plane of the series

$$
\zeta(z, q)=\sum_{n=0}^{\infty} \frac{1}{(n+q)^{z}}
$$

valid for $\operatorname{Re} z>1$ and $q \neq 0,-1,-2, \ldots$ The resulting function is analytic everywhere except at $z=1$, where it has a simple pole with unit residue.

For $q \neq 0$ one has

$$
\zeta(z, q+1)=\zeta(z, q)-\frac{1}{q^{z}}
$$

which, iterated $N$ times, leads to

$$
\sum_{n=0}^{N} \frac{1}{(n+q)^{z}}=\zeta(z, q)-\zeta(z, q+N+1) .
$$

This finite sum is what appears in the grand potential as a sum over Landau levels, and its expression in terms of Hurwitz Zeta functions is what allows us to expand the thermodynamic quantities in different limiting scenarios.

The derivative of $\zeta(z, q)$ with respect to $q$ is again a Hurwitz zeta function:

$$
\frac{\partial}{\partial q} \zeta(z, q)=-z \zeta(z+1, q)
$$

Hermite's integral representation 16

$$
\zeta(z, q)=\frac{1}{z-1} q^{-z+1}+\frac{1}{2} q^{-z}+2 q^{-z+1} \int_{0}^{\infty} \frac{\sin \left(z \tan ^{-1} t\right) d t}{\left(1+t^{2}\right)^{z / 2}\left(e^{2 \pi t q}-1\right)},(
$$

valid for all $z \neq 1$ and $q>0$, can be used to study the large $q$ behavior of $\zeta(z, q)$. For large $q$, the leading contribution to the integral in Eq. (63) comes from the region of small $t$. To isolate this contribution we split the integration range into $[0, a]$ and $[a, \infty)$, where $a$ is a fixed number less than one (for instance, $a=1 / 2$ ). In order to approximate the first integral we shall use the remarkable series expansion:

$$
\left(1+t^{2}\right)^{-z / 2} \sin \left(z \tan ^{-1}(t)\right)=\sum_{k=0}^{\infty}(-1)^{k} \frac{(z)_{2 k+1}}{(2 k+1) !} t^{2 k+1}
$$


which converges uniformly for $|t|<1$. In (64) $(z)_{n}$ is the Pochhammer symbol, or shifted factorial, defined by

$$
(z)_{n}=\frac{\Gamma(z+n)}{\Gamma(z)}=z(z+1) \cdots(z+n-1) .
$$

Thus, for a given $z$ and $0<t \leq a<1$ we have the uniform approximation

$$
\left|\frac{\sin \left(z \tan ^{-1} t\right)}{t\left(1+t^{2}\right)^{z / 2}}-\sum_{k=0}^{N}(-1)^{k} \frac{(z)_{2 k+1}}{(2 k+1) !} t^{2 k}\right|<\varepsilon,
$$

where $\varepsilon$ can be an arbitrarily small positive number and $N=N(\varepsilon, z)$ is a sufficiently big number. So we can write

$$
\int_{0}^{a} \frac{\sin \left(z \tan ^{-1} t\right)}{\left(1+t^{2}\right)^{z / 2}\left(e^{2 \pi q t}-1\right)} d t=\sum_{k=0}^{N}(-1)^{k} \frac{(z)_{2 k+1}}{(2 k+1) !} \int_{0}^{a} \frac{t^{2 k+1}}{e^{2 \pi q t}-1} d t+R(\varepsilon),
$$

with

$$
|R(\varepsilon)|<\int_{0}^{a} \frac{\varepsilon t}{e^{2 \pi q t}-1} d t<\varepsilon \int_{0}^{\infty} \frac{t}{e^{2 \pi q t}-1} d t=\frac{\varepsilon}{24 q^{2}} .
$$

Also, up to a correction that vanishes exponentially as $q \rightarrow \infty$, we can approximate

$$
\int_{0}^{a} \frac{t^{2 k+1}}{e^{2 \pi q t}-1} d t \simeq \int_{0}^{\infty} \frac{t^{2 k+1}}{e^{2 \pi q t}-1} d t=(-1)^{k} \frac{B_{2 k+2}}{4(k+1) q^{2 k+2}},
$$

where we have used formula 3.411.2. of reference [18]. The $B_{n}$ in $(69)$ are the Bernoulli numbers of even index, $B_{0}=1, B_{2}=1 / 6, B_{4}=-1 / 30$, etc. Since the integral over $[a, \infty)$ in (63) also gives a exponentially small contribution for large $q$, we finally have the result

$$
\begin{aligned}
\zeta(z, q)= & \frac{1}{z-1} q^{-z+1}+\frac{1}{2} q^{-z}+q^{-z-1} \sum_{k=0}^{N}(-1)^{k} \frac{(z)_{2 k+1} B_{2 k+2}}{(2 k+2) ! q^{2 k}} \\
& +O(\varepsilon)+O\left(e^{-\alpha q}\right) .
\end{aligned}
$$

Letting $\varepsilon \rightarrow 0$ (and consequently $N \rightarrow \infty$ ) we obtain the following expansion for $\zeta(z, q)$ :

$$
\begin{aligned}
\zeta(z, q) & =\frac{1}{z-1} q^{1-z}+\frac{1}{2} q^{-z}+\sum_{k=0}^{\infty} \frac{B_{2 k+2}}{(2 k+2) !}(z)_{2 k+1} \frac{1}{q^{z+2 k+1}} \\
& =\frac{1}{\Gamma(z)} \sum_{k=0}^{\infty}(-1)^{k} \frac{B_{k}}{k !} \frac{\Gamma(k+z-1)}{q^{k+z-1}},
\end{aligned}
$$

where (72) follows because the only non-vanishing Bernoulli number of odd index is $B_{1}=-1 / 2$. 
The cases $z=-1 / 2$ and $z=1 / 2$ are of special relevance for the work of this paper and we give the corresponding asymptotic expansions explicitly:

$$
\begin{aligned}
\zeta\left(-\frac{1}{2}, q\right) & =-\frac{2}{3} q^{3 / 2}+\frac{1}{2} q^{1 / 2}-\frac{1}{24} q^{-1 / 2}+O\left(q^{-5 / 2}\right) \\
\zeta\left(\frac{1}{2}, q\right) & =-2 q^{1 / 2}+\frac{1}{2} q^{-1 / 2}+\frac{1}{24} q^{-3 / 2}+O\left(q^{-7 / 2}\right) .
\end{aligned}
$$

For general $z$, the expansion $(71)$ is only asymptotic. It is easy to check that its radius of convergence, as a series in the variable $1 / q$, is zero. However, as an aside we should mention that for $z=-m$, where $m$ is a non-negative integer, the series (71) terminates, in view of $(-m)_{n}=0$ for $n>m$. Hence the series becomes a finite polynomial in $q$, actually a Bernoulli polynomial, up to a multiplicative constant:

$$
\zeta(-m, q)=-\frac{1}{m+1} B_{m+1}(q), \quad m=0,1,2, \cdots .
$$

The Hurwitz zeta function for $z<0$ admits the following Fourier expansion in the range $0<q<1$ :

$$
\zeta(z, q)=\frac{2 \Gamma(1-z)}{(2 \pi)^{1-z}} \times\left(\sin \left(\frac{\pi z}{2}\right) \sum_{n=1}^{\infty} \frac{\cos (2 \pi q n)}{n^{1-z}}+\cos \left(\frac{\pi z}{2}\right) \sum_{n=1}^{\infty} \frac{\sin (2 \pi q n)}{n^{1-z}}\right) .
$$

\section{Appendix B: Finite Temperature from $\mathrm{T}=0$}

In this appendix we show that the full finite temperature grand potential shown in Eq. (21) can be obtained from its zero temperature limit as

$$
\Omega(T, \mu)=\int_{-\frac{\mu-m}{T}}^{\infty} \Omega_{0}(\mu+T x) h(x) d x .
$$

where $h(x)$ is the Fermi-Dirac hump,

$$
h(x)=\frac{e^{x}}{\left(e^{x}+1\right)^{2}}=\frac{1}{4 \cosh ^{2}(x / 2)} .
$$

Actually, result (77) is a special case of the fact that any physical quantity of the form

$$
Q(T, \mu)=\int_{m}^{\infty} q(E) \frac{1}{e^{(E-\mu) / T}+1} d E
$$

can be computed in terms of its zero temperature limit $Q_{0}(\mu)$ as

$$
Q(T, \mu)=\int_{-\frac{\mu-m}{T}}^{\infty} Q_{0}(\mu+T x) h(x) d x .
$$


To prove $(80)$ we express the function $q(E)$ in Eq. (79) as the derivative of a function $F(E)$ defined as:

$$
F(E) \equiv \int_{m}^{E} q\left(E^{\prime}\right) d E^{\prime}
$$

and integrate by parts. After the change of variable $E=\mu+T x$ we obtain

$$
Q(T, \mu)=\int_{-\frac{\mu-m}{T}}^{\infty} F(\mu+T x) \frac{e^{x}}{\left(e^{x}+1\right)^{2}} d x .
$$

But from (79) it is seen that the function $F(\mu)$ is precisely the value of $Q(T, \mu)$ in the limit $T \rightarrow 0$ :

$$
Q_{0}(\mu)=\lim _{T \rightarrow 0} Q(T, \mu)=\int_{m}^{\mu} q(E) d E \equiv F(\mu) .
$$

This establishes (80). Now, to prove Eq. (77) for the grand potential, we merely notice that the standard expression for it shown in Eq. (21) can be turned into the form (79) by expressing the density of states $g(E)$ as the derivative of the function $G(E)$-which is the total number of single particle states up to energy E:

$$
G(E)=\int_{m}^{E} g\left(E^{\prime}\right) d E^{\prime}
$$

and then integrating by parts to obtain

$$
\Omega(T, \mu)=-\int_{m}^{\infty} G(E) \frac{1}{e^{(E-\mu) / T}+1} d E .
$$

This expression for the grand potential is precisely of the form shown in Eq. (79), with $q(E)=-G(E)$.

In the degenerate regime, $T \ll \mu-m$, the lower limit of integration in (77) can be replaced by $-\infty$ with negligible error. Additionally, if the function $\Omega_{0}(\mu+T x)$ varies slowly under the hump (this may not be the case for the oscillatory terms; see section 5), then it can be expanded in a Taylor series around $\mu$ and the resulting terms integrated one by one. Using now the results

$$
\begin{gathered}
\int_{-\infty}^{\infty} h(x) d x=1, \quad \int_{-\infty}^{\infty} x^{2} h(x) d x=\frac{\pi^{2}}{3}, \quad \int_{-\infty}^{\infty} x^{4} h(x) d x=\frac{7 \pi^{4}}{15} \\
\quad \ldots \int_{-\infty}^{\infty} x^{2 n} h(x) d x=\pi^{2 n}\left|\left(2^{2 n}-2\right) B_{2 n}\right|
\end{gathered}
$$

we obtain the small temperature expansion,

$$
\Omega(T, \mu)=\Omega_{0}(\mu)+\frac{\pi^{2}}{6} T^{2} \Omega_{0}^{\prime \prime}(\mu)+\frac{7 \pi^{4}}{360} T^{4} \Omega_{0}^{(4)}(\mu) \ldots
$$




\section{Appendix C: Reduction of the Oscillatory Con- tribution}

Let $p(q)$ be a function in the unit interval $[0,1]$ periodically extended over the real axis, and $f(q)$ an arbitrary function. Then, the integral of the product of these functions over an arbitrary interval can be separated into a sum of integrals over integer intervals, plus a residual integral, as follows:

$$
\int_{0}^{Q} p(q) f(q) d q=\int_{0}^{1} p(q) \sum_{k=0}^{\lfloor Q\rfloor-1} f(q+k) d q+\int_{0}^{\{Q\}} p(q) f(q+\lfloor Q\rfloor) d q .
$$

We now specialize this result for $p(q)=\zeta(z,\{q\})$ as the periodic function, $f(q)=(1+b q)^{-s}$ and $Q=p_{\mathrm{F}}^{2} / b$, and use Eq. (61) to express the sum in terms of Hurwitz functions:

$$
\begin{aligned}
\sum_{k=0}^{\left\lfloor p_{\mathrm{F}}^{2} / b\right\rfloor-1} \frac{1}{[1+b(q+k)]^{s}} & =\frac{1}{b^{s}} \sum_{k=0}^{\left\lfloor p_{\mathrm{F}}^{2} / b\right\rfloor-1} \frac{1}{[(q+1 / b)+k]^{s}} \\
& =\frac{1}{b^{s}}\left[\zeta(s, q+1 / b)-\zeta\left(s, q+1 / b+\left\lfloor p_{\mathrm{F}}^{2} / b\right\rfloor\right)\right] .
\end{aligned}
$$

We thus find the integral expression

$$
\begin{aligned}
\int_{0}^{p_{\mathrm{F}}^{2} / b} \frac{\zeta(z,\{q\})}{(1+b q)^{s}} d q & =\frac{1}{b^{s}}\left\{\int_{0}^{\left\{p_{\mathrm{F}}^{2} / b\right\}} \frac{\zeta(z, q)}{\left(q+1 / b+\left\lfloor p_{\mathrm{F}}^{2} / b\right\rfloor\right)^{s}} d q\right. \\
& \left.+\int_{0}^{1} \zeta(z, q)\left[\zeta(s, q+1 / b)-\zeta\left(s, q+1 / b+\left\lfloor p_{\mathrm{F}}^{2} / b\right\rfloor\right)\right] d q\right\}
\end{aligned}
$$

Using this generic result one derives the expression given in Eq. (36) for the oscillatory piece of the grand potential.

\section{Appendix D: Small $b$ Expansions}

In this appendix we show that

$$
\begin{gathered}
-\int_{0}^{p_{\mathrm{F}}^{2} / b} \frac{\tilde{\zeta}_{3}\left(-\frac{1}{2}, q+1\right)}{\sqrt{1+b q}} d q=\frac{2}{3} \zeta\left(-\frac{3}{2}\right)+\frac{1}{60}+b\left[\frac{2}{15} \zeta\left(-\frac{5}{2}\right)-\frac{1}{1260}\right] \\
+O\left(b^{3 / 2}\right) \ldots
\end{gathered}
$$

and

$$
\begin{aligned}
\int_{0}^{p_{\mathrm{F}}^{2} / b} \frac{\zeta\left(-\frac{1}{2},\{q\}\right)}{\sqrt{1+b q}} d q= & -\frac{2}{3} \zeta\left(-\frac{3}{2}\right)-\frac{2}{15} \zeta\left(-\frac{5}{2}\right) b-\frac{2}{35} \zeta\left(-\frac{7}{2}\right) b^{2} \\
& +\frac{2}{3} \frac{1}{\varepsilon_{\mathrm{F}}} \zeta\left(-\frac{3}{2},\left\{p_{\mathrm{F}}^{2} / b\right\}\right)+\frac{2}{15} \frac{b}{\varepsilon_{\mathrm{F}}^{3}} \zeta\left(-\frac{5}{2},\left\{p_{\mathrm{F}}^{2} / b\right\}\right) \\
& +\frac{2}{35} \frac{b^{2}}{\varepsilon_{\mathrm{F}}^{5}} \zeta\left(-\frac{7}{2},\left\{p_{\mathrm{F}}^{2} / b\right\}\right)+O\left(b^{3}\right) \ldots
\end{aligned}
$$


The first expansion is needed in order to find the small $b$ behavior of the nonoscillatory piece of the grand potential, Eq. (34), and the second expansion is required for the oscillatory piece, which is exhibited in Eq. (36) already making use of the formula (88).

To prove Eq. (89), we first define the integral

$$
R(b) \equiv-\int_{0}^{p_{\mathrm{F}}^{2} / b} \frac{\tilde{\zeta}_{3}\left(-\frac{1}{2}, q+1\right)}{\sqrt{1+b q}} d q,
$$

and then try to expand it in powers of $b$. The first two terms of the expansion are easily found, using the results:

$$
\begin{aligned}
\left.R(b)\right|_{b=0} & =\frac{2}{3} \zeta\left(-\frac{3}{2}\right)+\frac{1}{60}, \\
\left.\frac{\partial}{\partial b} R(b)\right|_{b=0} & =\frac{2}{15} \zeta\left(-\frac{5}{2}\right)-\frac{1}{1260},
\end{aligned}
$$

which follow from the facts that $\tilde{\zeta}_{3}\left(-\frac{1}{2}, q+1\right)$ decreases like $q^{-5 / 2}$ when $q \rightarrow \infty$, and that the antiderivative of $\tilde{\zeta}_{3}\left(-\frac{1}{2}, q\right)$, which is $\frac{2}{3} \tilde{\zeta}_{3}\left(-\frac{3}{2}, q\right)$, vanishes like $q^{-3 / 2}$ when $q \rightarrow \infty$. Indeed, we can directly calculate at $b=0$ :

$$
\begin{aligned}
\left.R(b)\right|_{b=0}=-\int_{1}^{\infty} \tilde{\zeta}_{3}\left(-\frac{1}{2}, q\right) d q & =\frac{2}{3} \tilde{\zeta}_{3}\left(-\frac{3}{2}, 1\right) \\
& =\frac{2}{3} \zeta\left(-\frac{3}{2}\right)+\frac{1}{60},
\end{aligned}
$$

and also

$$
\begin{aligned}
\left.\frac{\partial}{\partial b} R(b)\right|_{b=0}= & \lim _{b \rightarrow 0}\left[\tilde{\zeta}_{3}\left(-\frac{1}{2}, 1+p_{\mathrm{F}}^{2} / b\right) \frac{p_{\mathrm{F}}^{2}}{\varepsilon_{\mathrm{F}} b^{2}}\right. \\
& \left.\quad+\frac{1}{2} \int_{0}^{p_{\mathrm{F}}^{2} / b} \tilde{\zeta}_{3}\left(-\frac{1}{2}, q+1\right) \frac{q}{(1+b q)^{3 / 2}} d q\right] \\
= & \frac{1}{2} \int_{0}^{\infty} q \tilde{\zeta}_{3}\left(-\frac{1}{2}, q+1\right) d q .
\end{aligned}
$$

In this last expression, the $b \rightarrow 0$ limit of the first term inside the square bracket vanishes due to the large- $q$ behavior of $\tilde{\zeta}_{3}\left(-\frac{1}{2}, q\right)$, while the last integral can be done by parts:

$$
\begin{aligned}
\frac{1}{2} \int_{0}^{\infty} q \tilde{\zeta}_{3}\left(-\frac{1}{2}, q+1\right) d q & =\frac{1}{2}\left(-\frac{2}{3}\right) \int_{0}^{\infty} \tilde{\zeta}_{3}\left(-\frac{3}{2}, q+1\right) d q \\
& =\frac{1}{3} \cdot \frac{2}{5} \tilde{\zeta}_{3}\left(-\frac{5}{2}, 1\right)=\frac{2}{15} \zeta\left(-\frac{5}{2}\right)-\frac{1}{1260}
\end{aligned}
$$

We should point out that the next term in the expansion (89) is not $O\left(b^{2}\right)$, as it would be in a regular Taylor series, because $R^{\prime \prime}(0)$ does not exist. To get this 
singular term one should go back to the original definition of $R(b)$ and subtract the leading term in the asymptotic expansion of $\tilde{\zeta}_{3}\left(-\frac{1}{2}, q\right)$ :

$$
\tilde{\zeta}_{3}\left(-\frac{1}{2}, q\right)=\frac{1}{1920 q^{5 / 2}}+\tilde{\zeta}_{4}\left(-\frac{1}{2}, q\right),
$$

where $\tilde{\zeta}_{4}\left(-\frac{1}{2}, q\right)$ is of order $O\left(q^{-9 / 2}\right)$. Then, the resulting term in the integrand, $(1+q)^{-5 / 2}(1+b q)^{-1 / 2}$, can be integrated explicitly, and its small $b$ expansion reads

$$
\int_{0}^{p_{\mathrm{F}}^{2} / b} \frac{1}{(1+q)^{5 / 2} \sqrt{1+b q}} d q=\frac{2}{3}-\frac{2}{3} b+\frac{2}{3} \frac{\varepsilon_{\mathrm{F}}}{p_{\mathrm{F}}^{3}}\left(2 p_{\mathrm{F}}^{2}-1\right) b^{3 / 2}+O\left(b^{2}\right)
$$

Therefore, the $O\left(b^{3 / 2}\right)$ term in the expansion of $R(b)$ is actually

$$
-\frac{1}{2880} \frac{\varepsilon_{\mathrm{F}}}{p_{\mathrm{F}}^{3}}\left(2 p_{\mathrm{F}}^{2}-1\right) b^{3 / 2} .
$$

With this result we complete the proof of Eq. (89).

We now proceed to prove Eq. (90), which is relevant for the small $b$ expansion of the oscillatory piece of the grand potential. First we must realize that the integral in Eq. (90) is of the type studied in Appendix C, and in particular the result shown in Eq. (88) applies. We will then proceed to expand that result for small $b$. To expand the first integral in Eq. (88), we just need to use the binomial expansion of $\left(q+\frac{1}{b}+\left\lfloor p_{\mathrm{F}}^{2} / b\right\rfloor\right)^{-1 / 2}$, where $q \ll 1 / b+\left\lfloor p_{\mathrm{F}}^{2} / b\right\rfloor$, and then use the integrals:

$$
\begin{aligned}
\int_{0}^{Q} \zeta\left(-\frac{1}{2}, q\right) d q & =\frac{2}{3} \zeta\left(-\frac{3}{2}, Q\right)-\frac{2}{3} \zeta\left(-\frac{3}{2}\right) \\
\int_{0}^{Q} q \zeta\left(-\frac{1}{2}, q\right) d q & =-\frac{4}{15} \zeta\left(-\frac{5}{2}, Q\right)+\frac{4}{15} \zeta\left(-\frac{5}{2}\right)+\frac{2}{3} Q \zeta\left(-\frac{3}{2}, Q\right)
\end{aligned}
$$

We thus find the expansion for the first integral of Eq. (88):

$$
\begin{aligned}
& \int_{0}^{\left\{p_{\mathrm{F}}^{2} / b\right\}} \frac{\zeta\left(-\frac{1}{2}, q\right)}{\left(q+1 / b+\left\lfloor p_{\mathrm{F}}^{2} / b\right\rfloor\right)^{1 / 2}} d q \\
& =\frac{1}{\left(1+b\left\lfloor p_{\mathrm{F}}^{2} / b\right\rfloor\right)^{1 / 2}}\left[\frac{2}{3} \zeta\left(-\frac{3}{2},\left\{p_{\mathrm{F}}^{2} / b\right\}\right)-\frac{2}{3} \zeta\left(-\frac{3}{2}\right)\right] \\
& -\frac{b}{\left(1+b\left\lfloor p_{\mathrm{F}}^{2} / b\right\rfloor\right)^{3 / 2}}\left[-\frac{2}{15} \zeta\left(-\frac{5}{2},\left\{p_{\mathrm{F}}^{2} / b\right\}\right)+\frac{2}{15} \zeta\left(-\frac{5}{2}\right)\right. \\
& \left.\quad+\frac{1}{3}\left\{p_{\mathrm{F}}^{2} / b\right\} \zeta\left(-\frac{3}{2},\left\{p_{\mathrm{F}}^{2} / b\right\}\right)\right]+O\left(b^{2}\right) \ldots
\end{aligned}
$$

Notice that the terms of the form $\left(1+b\left\lfloor p_{\mathrm{F}}^{2} / b\right\rfloor\right)^{-n / 2} \zeta\left(-\frac{n+2}{2}\right)$ will cancel in the full oscillatory piece, Eq. (36), when expansions (98) and (102) (see below) 
are combined. For the remaining terms containing $\left(1+b\left\lfloor p_{\mathrm{F}}^{2} / b\right\rfloor\right)$ in the denominator we write $\left.1+b \mid p_{\mathrm{F}}^{2} / b\right\rfloor=\left(1+p_{\mathrm{F}}^{2}\right)-b\left\{p_{\mathrm{F}}^{2} / b\right\}$ and perform a binomial expansion, which leads to a further cancellation of all the terms of the type $\left\{p_{\mathrm{F}}^{2} / b\right\}^{k} \zeta\left(z,\left\{p_{\mathrm{F}}^{2} / b\right\}\right)$ with $k \geq 1$.

Now we need to expand the second integral in Eq. 887. In this case we must expand the $\zeta$ functions inside the squared brackets for large values of their argument. We thus use the large- $q$ expansions of $\zeta\left( \pm \frac{1}{2}, q\right)$ shown in Eqs. (73) and (74), together with the definite integrals [19]

$$
\begin{aligned}
\int_{0}^{1} \zeta\left(-\frac{1}{2}, q\right) d q & =0 \\
\int_{0}^{1} q \zeta\left(-\frac{1}{2}, q\right) d q & =\frac{2}{3} \zeta\left(-\frac{3}{2}\right) \\
\int_{0}^{1} q^{2} \zeta\left(-\frac{1}{2}, q\right) d q & =\frac{2}{3} \zeta\left(-\frac{3}{2}\right)-\frac{8}{15} \zeta\left(-\frac{5}{2}\right)
\end{aligned}
$$

We thus get the expansion:

$$
\begin{aligned}
& \frac{1}{\sqrt{b}} \int_{0}^{1} \zeta\left(-\frac{1}{2}, q\right)\left[\zeta\left(\frac{1}{2}, q+1 / b\right)-\zeta\left(\frac{1}{2}, q+1 / b+\left\lfloor p_{\mathrm{F}}^{2} / b\right\rfloor\right)\right] d q \\
& =-\frac{2}{3} \zeta\left(-\frac{3}{2}\right)\left[1-\frac{1}{\left(1+b\left\lfloor p_{\mathrm{F}}^{2} / b\right\rfloor\right)^{1 / 2}}\right]-\frac{2}{15} \zeta\left(-\frac{5}{2}\right)\left[1-\frac{1}{\left(1+b\left\lfloor p_{\mathrm{F}}^{2} / b\right\rfloor\right)^{3 / 2}}\right] b \\
& \quad+O\left(b^{2}\right) \ldots
\end{aligned}
$$

which completes the proof of Eq. (90) up to order $b$. The $O\left(b^{2}\right)$ terms are obtained in a similar fashion.

Finally, and for completeness, we also present here the small $b$ expansion of the explicit terms in (34), which reads

$$
\begin{aligned}
\frac{1}{2}\left(1-b+\frac{1}{6} b^{2}\right) \ln \left(\frac{\varepsilon_{\mathrm{F}}+\sqrt{b+p_{\mathrm{F}}^{2}}}{1+\sqrt{b}}\right)-\frac{1}{2}\left[\left(b+p_{\mathrm{F}}^{2}\right)^{1 / 2} \varepsilon_{\mathrm{F}}-b^{1 / 2}\right] \\
+\frac{1}{3}\left[\left(b+p_{\mathrm{F}}^{2}\right)^{3 / 2} \varepsilon_{\mathrm{F}}-b^{3 / 2}\right]+\frac{1}{2} b\left[\cosh ^{-1}\left(\varepsilon_{\mathrm{F}}\right)-p_{\mathrm{F}} \varepsilon_{\mathrm{F}}\right] \\
=\frac{1}{2} \cosh ^{-1}\left(\varepsilon_{\mathrm{F}}\right)-\frac{1}{2} p_{\mathrm{F}} \varepsilon_{\mathrm{F}}+\frac{1}{3} p_{\mathrm{F}}^{3} \varepsilon_{\mathrm{F}}+\frac{1}{12} b^{2} \cosh ^{-1}\left(\varepsilon_{\mathrm{F}}\right) \\
-\frac{1}{60} b^{5 / 2}+\frac{1}{1260} b^{7 / 2}+O\left(b^{9 / 2}\right) \ldots
\end{aligned}
$$

One should notice that all the terms that are independent of the Fermi energy (i.e. $p_{\mathrm{F}}$ or $\varepsilon_{\mathrm{F}}$ ) are spurious, cancelling between the different expansions and thus leading to result (38). 


\section{References}

[1] V. Canuto and H. Chiu, Phys. Rev. 173, 1210 (1968); ibid. 173, 1220 (1968); ibid. 173, 1229 (1968); H.J. Lee, V. Canuto, H. Chiu and C. Chiuderi, Phys. Rev. Lett. 23, 390 (1969); V. Canuto, H. Chiu and C. Chiuderi, Nature 225, 47 (1970).

[2] R.F. O’Connell and K.M. Roussel, Astron. \& Astroph. 18, 198 (1972).

[3] J. Schmid-Burgk, Astron. \& Astroph. 26, 335 (1973).

[4] R.D. Blanford and L. Hernquist, J. Phys. C15, 6233 (1982).

[5] A. Chodos, K. Everding and D. Owen, Phys. Rev. D42, 2881 (1990).

[6] S.K. Blau, M. Visser and A. Wipf, Intl. Jour. Mod. Phys. A6 5409 (1991).

[7] P. Elmfors, D. Persson and B-S. Skagerstam, Phys. Rev. Lett. 71, 480 (1993); Astropart. Phys. 2, 299 (1994).

[8] Vad. Zeitlin, hep-ph/9412204 (unpublished); D. Persson and Vad. Zeitlin, Phys. Rev. D51, 2026 (1995); Vad. Zeitlin, J. Exp. Theor. Phys. 82, 79 (1996).

[9] D. Cangemi and G. Dunne, Annals Phys. 249, 582 (1996).

[10] L. D. Landau, Zeitschrift für Physik 64, 629 (1930).

[11] L. D. Landau, Proc. Royal Soc. of London, A170, 363 (1939).

[12] See, for example, L.D. Landau and E.M. Lifshitz, Satistical Physics, Part I., 3rd. Edition, Pergamon Press (1980).

[13] M.H. Johnson and B.A. Lippmann, Phys. Rev. 76, 828 (1949); H. Robl Acta Phys. Austriaca 6, 105 (1952).

[14] L.D. Landau and E.M. Lifshitz, Quantum Mechanics (Non-relativistic Theory). Course of Theoretical Physics, volume 3, third edition. Pergamon Press, 1977.

[15] Notice that $B_{0}$ is in our case half of the scale used by other authors [1, 4. $\left(m^{2} c^{3} / e \hbar \approx 4.4 \times 10^{13} \mathrm{G}\right)$, because the natural dimensionless variable associated to the magnetic field in this problem is $b=B / B_{0}=2 e B / m^{2}$, as seen in Eqs. (1, 2).

[16] E. Whittaker and G. Watson, A course of Modern Analysis, Cambridge University Press, Fourth Edition reprinted, 1963.

[17] J. Spanier and K.B. Oldham, An Atlas of Functions, Hemisphere Publishing Corp., 1987. 
[18] I.S. Gradshteyn and I.M. Ryzhik, Table of Integrals, Series and Products, fifth edition, ed. Alan Jeffrey. Academic Press, 1994.

[19] O. Espinosa and V. Moll, On some definite integrals involving the Hurwitz Zeta function, April 2000, to appear in The Ramanujan Journal. 


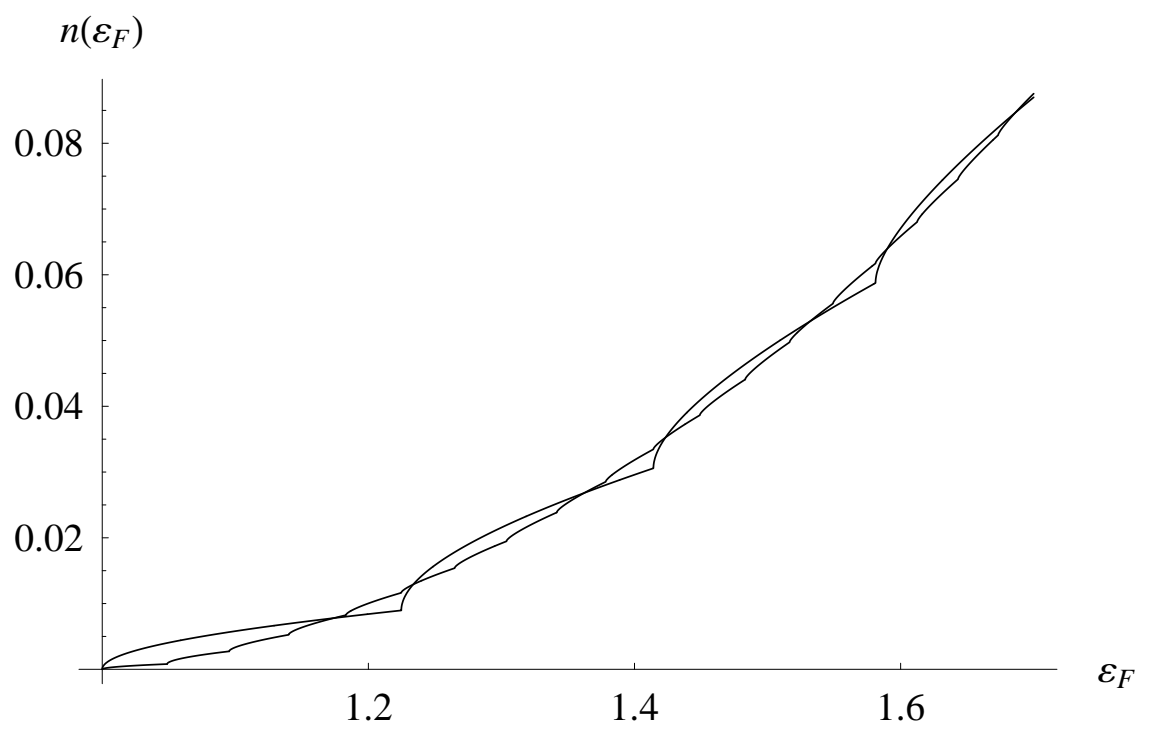

Figure 1: The particle density at $\mathrm{T}=0$ as a function of the Fermi energy, for fixed magnetic field $b=0.1$ (smooth curve) and $b=0.5$ (bumpy curve). 


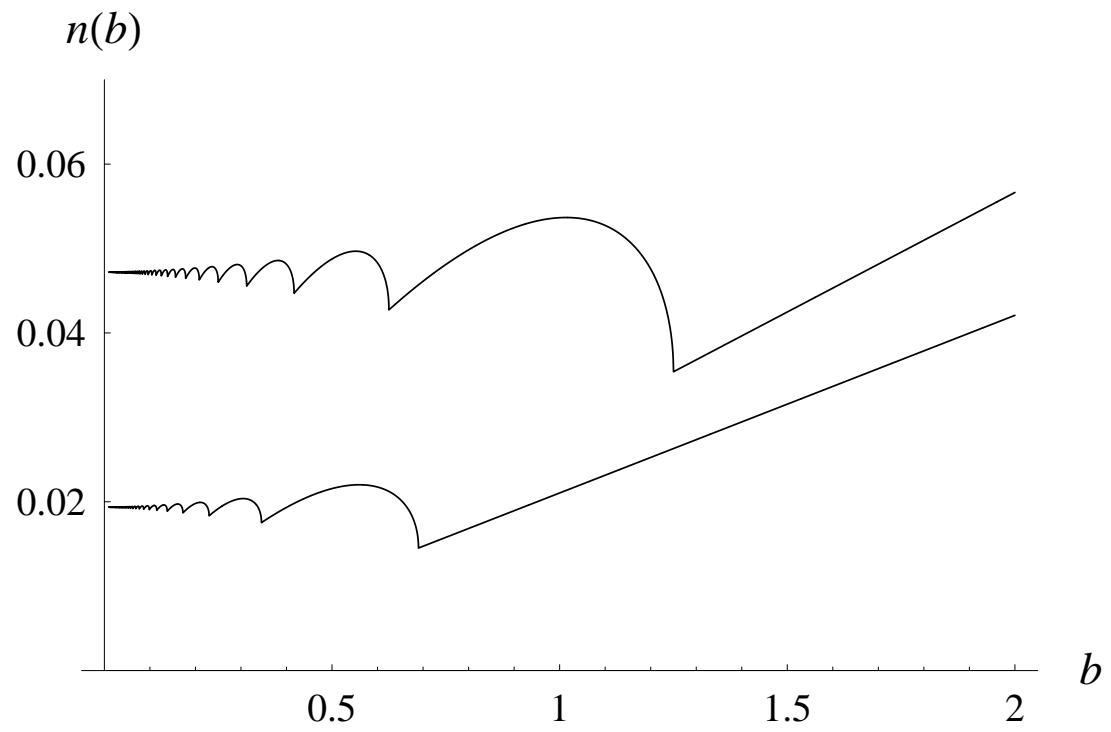

Figure 2: The particle density at $\mathrm{T}=0$ as a function of the magnetic field, for fixed Fermi energy $\varepsilon_{\mathrm{F}}=1.3$ (lower curve) and $\varepsilon_{\mathrm{F}}=1.5$ (upper curve). 


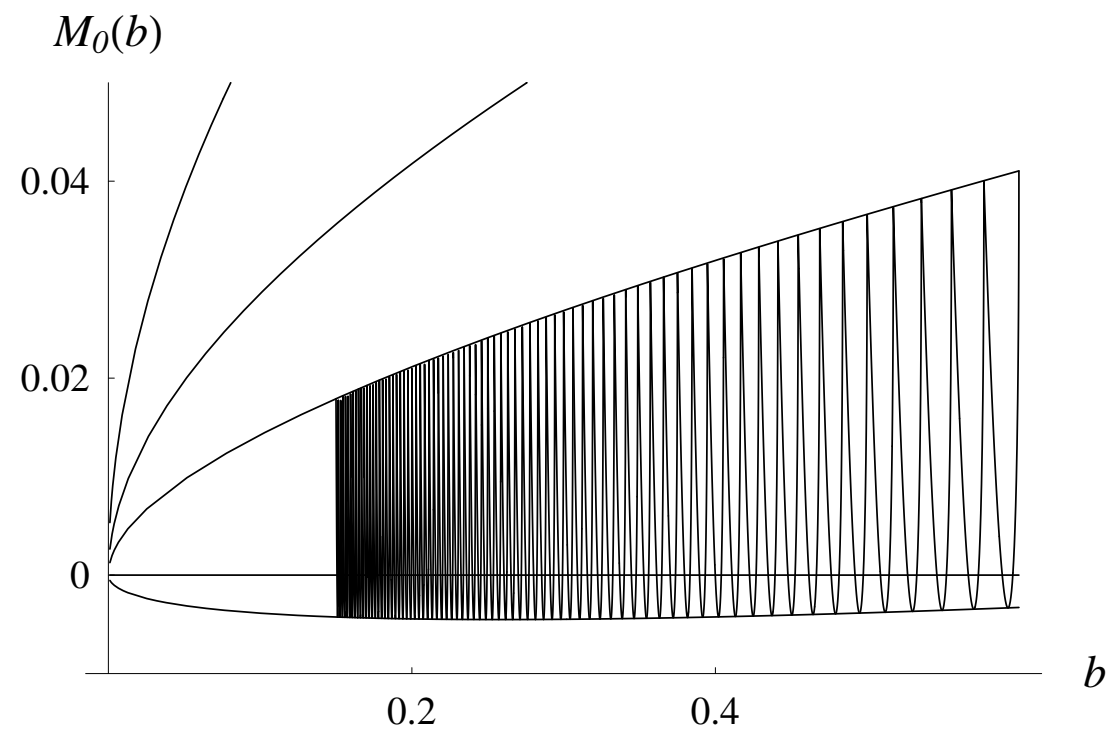

Figure 3: The magnetization at $T=0$ which oscillates as a function of the magnetic field $b$, for Fermi energy $\varepsilon_{\mathrm{F}}=4$. Also shown are the upper and lower envelopes of the curve, and the upper envelopes for larger Fermi energies $\left(\varepsilon_{\mathrm{F}}=8\right.$ and 16). 


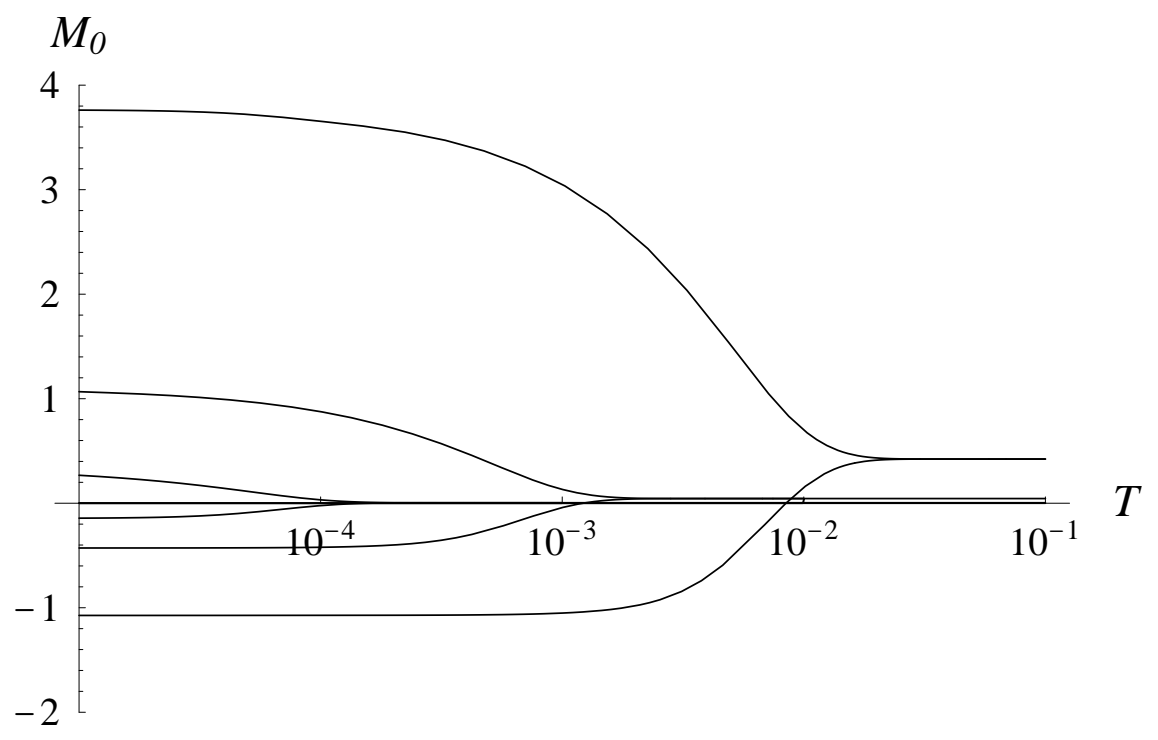

Figure 4: The upper envelope of the magnetization as a function of the temperature, for fixed chemical potential $\mu=100$, and magnetic field $b=0.1,1$ and 10 (lower, medium and upper curve, respectively). 


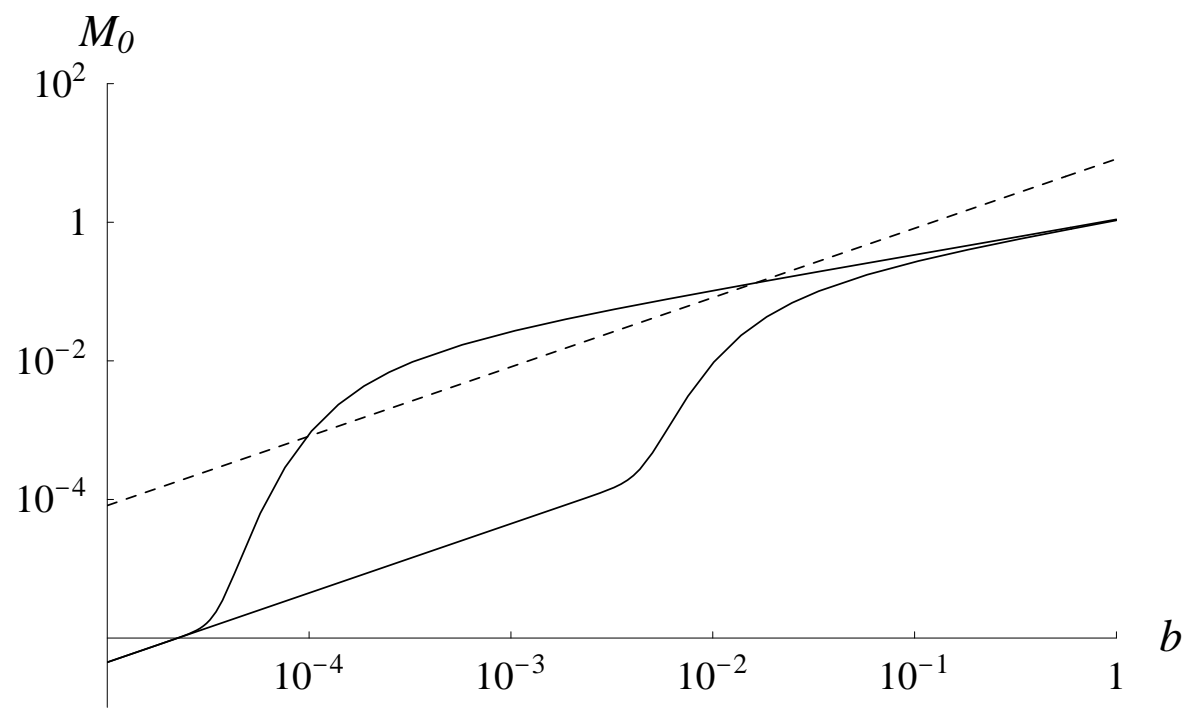

Figure 5: The upper envelope of the magnetization as a function of the magnetic field $b$, for two temperatures $T=10^{-5}$ and $10^{-7}$ (lower and upper curve, respectively). The chemical potential is fixed at $\mu=100$. The dashed line represents the macroscopic relation $M=\frac{3}{8 \pi} B$, for a uniformly magnetized sphere. 


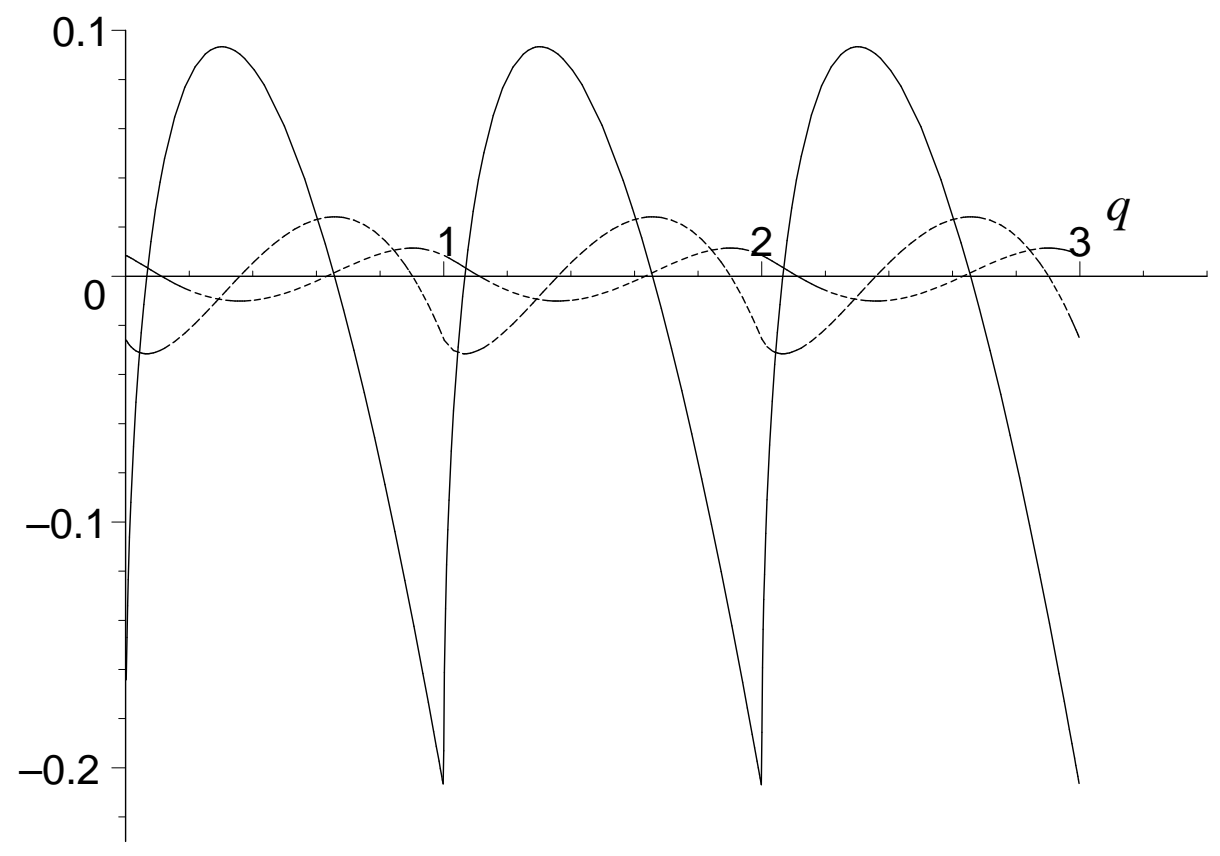

Figure 6: The functions $\zeta(z,\{q\})$, for $z=-1 / 2,-3 / 2,-5 / 2$ (large, medium and small amplitude, respectively). 